\title{
PLASMA TRANSPORT IN STOCHASTIC MAGNETIC FIELDS。 I I GENERAL CONSIDERATIONS AND TEST PARTICLE TRANSPORT
}

\section{BY}

J. A, KROMMES, R, G, KLEVA, AND $C$. OBERMAN

\section{PLASMA PHYSICS LABORATORY}

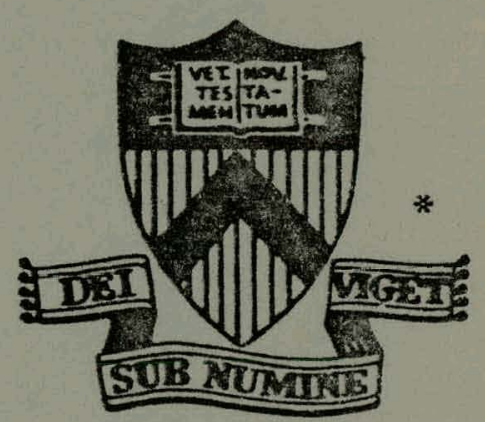

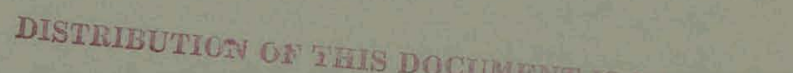

\section{PRINCETON \\ UNIVERSITY PRINCETON, NEW JERSEY}

This work was supported by the U. S. Department of Energy Contract No. EY-76-C-02-3073. Reproduction, translation, publication, use and disposal, in whole or in part, by or for the United States Government is permitted. 


\section{DISCLAIMER}

This report was prepared as an account of work sponsored by an agency of the United States Government. Neither the United States Government nor any agency Thereof, nor any of their employees, makes any warranty, express or implied, or assumes any legal liability or responsibility for the accuracy, completeness, or usefulness of any information, apparatus, product, or process disclosed, or represents that its use would not infringe privately owned rights. Reference herein to any specific commercial product, process, or service by trade name, trademark, manufacturer, or otherwise does not necessarily constitute or imply its endorsement, recommendation, or favoring by the United States Government or any agency thereof. The views and opinions of authors expressed herein do not necessarily state or reflect those of the United States Government or any agency thereof. 


\section{DISCLAIMER}

Portions of this document may be illegible in electronic image products. Images are produced from the best available original document. 
This report was prepared as an account of work sponsored by the United States Government. Neither the United States nor the United States Energy Research and Development Administration, nor any of their employees, nor any of their contractors, subcontractors, or their employees, makes any warranty, express or implied, or assumes any legal liability or responsibility for the accuracy, completeness or usefulness of any information, apparatus, product or process disclosed, or represents that its use would not infringe privately owned rights.

Printed in the United States of America.

Available from

National Technical Information Service

U. S. Department of Commerce 5285 port Royal Road

Springfield, Virginia 22151

Price: Printed Copy $\$+\star$; Microfiche $\$ 3.00$

*Pages

$1-50$

$51-150$

$151-325$

$326-500$

$501-1000$
NTIS

Selling Price

$\$ 4.00$

5.45

7.60

10.60

13.60 


\begin{abstract}
$p^{20}$ tor.
Plasma Transport in Stochastic Magnetic Fieldse

General Considerations and Test Particle Transport
\end{abstract}

John A. Krommes, Robert G. Kleva, and Carl Oberman

Plasma Physics Laboratory, Princeton University

Princeton, New Jersey 08540 USA

A systematic theory is developed for the

computation of electron transport in stochastic

magnetic fields. Small scale magnetic perturba-

tions arising, for example, from finite- $\beta$ micro-

instabilities are assumed to destroy the flux

surfaces of a standard tokamak equilibrium.

Because the magnetic lines then wander in a volume,

electron radial flux is enhanced due to the rapid

particle transport along as well as across the lines.

By treating the magnetic Iines as random variables,

it is possible to develop a kinetic equation for

the electron distribution function. This is solved

approximately to yield the diffusion coefficient. 
1. Introduction

Stochasticity of magnetic lines affords a likely explanation for the enhanced electron heat conduction observed in tokamaks (TFR Group 1977). The mechanism, proposed by Stix (1973) and others (Rechester \& Rosenbluth 1978), involves the destruction of magnetic flux surfaces (Rosenbluth, Sagdeev, Taylor \& Zaslavskii 1966) by small scale radial magnetic perturbations resonant with unperturbed rational surfaccs, the consequent radial diffusion of magnetic lines, and the asanriated radial particlc flux due to particle transport predominantly along the Iines. The purpose of our research, which is by no means complete, is to present a systematic theory for electron particle transport and heat conduction, valid in principle for rather general stochastic magnetic fields. From such a theory one can hope to derive, hy systematic subsidiary approximations, results appropriate to practical situations of current interest such as the anomalous transport due to unstable drift-tearing modes (Chen, Rutherford \& Tang 1977) or other finite- $\beta$ fluctuations in tokamaks.

In the present paper we introduce the formalism and apply it to the simplest nontrivial problem: the radial diffusion of test electrons in magnetic fields which are specified statistically, not related self-consistently to the random plasma currents. One can infer from random walk arguments that, except for numerical 
factors of order unity, the electron thermal conduction will obey the same law. The macroscopic diffusion of the plasma fluid will be limited by ambipolar constraints, involving the ion response, which we do not discuss here.

A self-consistent description is necessary, of course, to describe the nonlinear evolution of the microinstabilities responsible for the magnetic fluctuations. It is noteworthy that our formalism embraces this possibility naturally. We shall, however, defer detailed treatment of the self-consistent problem to a future paper.

Recently, Rechester and Rosenbluth (RR) (Rechester \& Rosenbluth 1978) proposed scaling laws for heat transport in specified stochastic fields by using random walk arguments and certain intuitive notions of stochasticity theory (Chirikov 1969; Zaslavskii \& Chirikov 1971). Though that work is fundamental, important questions remained unanswered. Indeed, RR did not describe the region of parameter space -- in particular, the classes of stochastic fields -- to which their theory was restricted. One does not expect such a theory to be universal; without knowledge of validity restrictions, it is impossible to convincingly estimate transport coefficients for realistic devices. RR did not provide a formula for an important correlation or mixing length, which both determined the collisionality regime and appeared in the collisional result. They did not discuss the relation of their approach to the conventional plasma kinetic theories, though one expects that such a relation exists. Finally, it appcars difficult, if not impossible, to extend the approach of $R R$ to 
the fully self-consistent problem. The formalism which we develop allows natural resolution of all these questions. In particular, the scaling laws of $R R$ emerge as qualitative approximations well motivated in the limits of weak radial localization of the fluctuations and of sufficiently weak stochasticity. (We define these concepts precisely in Secs. $3,4$.$) we treat as well other$ regimes of interest. Unlike the technique of RR, our formalism is capable of predicting numerical coefficients. However, we have not fully explored this aspect of the theory.

The most important philosophical problem one must resolve in a transport study of this kind is concerned with the validity of the use of statistical techniques to describe the deterministic nature of magnetic lines. (If the magnetic fluctuations arise from turbulent fluctuations of the plasma, then the lines are, strictiy speaking, not deterministic. This is, however, a separate issue. Furthermore, the questions relating to determinism remain relevant when the background correlations decay sliswly, a useful approximation.) The fact that it is appropriate to use statistical argument's to describe the lines follows, in part, from deep theorems of topology far beyond the scope of this paper and, in part, from experience acquired from -numerical experiments on model systems. Both of these aspects assure us that the phase space of the lines is incredibly complicated, including elements of both coherence and randomness. Previous attempts at the analytic description of transport in such situations have essentially ignored these complicated details and replaced them by certain intuitive statistical assumptions. Because such approaches are generally tractable, 
they provide useful direction. However, predictions derived from these are useless, in a certain sense, unless the intuitive assumptions can be justified systematically. The purpose of this paper is, in part, to provide such justification. In doing so, we are led naturally to a more complete description than has been given previously.

The plan of the paper is as follows. In Sec. 2 we review some basic notions of diffusion and sketch the basic physics of particle transport in stochastic fields. In Sec. 3 we collect and discuss the various parameters which enter our systematic work. Section 4 contains a new, detailed heuristic derivation of the test electron diffusion coefficient. In Sec. 5 we reconsider the problem from the point of view of a kinetic theory for the distribution function of test electrons. This approach has the distinct advantage of being closely related to recent developments in fluid and plasma turbulence theory. In particular, we find interesting parallels between the microtopology of magnetic lines and the fine-scale granulations of weakly turbulent vlasov plasma. We discuss our conclusions in Sec. 6. Appendix A contains some details of the parallel motion of particles along lines.' In Appendix $B$ we review aspects of renormalized perturbation theory, an approach alternative to the nonperturbative one we adopt in the text. 
2. Qualitative Physics of Stochastic Transport

In this section we give a descriptive introduction to the problem of particle transport in stochastic magnetic fiélds and summarize qualitatively our principle results. We ignore toroidal effects and consider a standard sheared tokamak field, with concentric circular flux surfaces, perturbed by a small radial perturbation $B_{r}$. Thus, $\underset{\sim}{B}=\left(B_{r}, B_{\theta}, B_{\phi}\right)=(b, \varepsilon / q, 1) B_{0}$, where $\varepsilon \equiv r / R<1$ is the inverse aspect ratio, $q(r)$ is the safety factor, $\theta$ and $\phi$ are the poloidal and toroidal angles, $B_{0}$ is taken to be constant, and if $\mu$ denotes the set $\{m, n\}$ of poloidal (m) and toroidal ( $n$ ) mode numbers, then

$$
b \equiv B_{r} / B_{0}=\sum_{\mu} b_{\mu}(r, t) \exp [i(m \theta-n \phi)]
$$

When the Fourier amplitudes $b_{\mu}$ have radial dependence, we strictly require small corrections to $B_{\theta}$ and $B_{\phi}$ to maintain $\underset{\sim}{\nabla} \cdot \underset{\sim}{B}=0$. Since we assume $B_{r} \ll B_{\dot{\theta}}$, these corrections; are unimportant. If only one mode $\left(b_{\mu}+c_{.} c\right)$ is excited, flux surfaces persist (mathematically, the system remains integrable), with the topology however altered to include a chain of magnetic islands of width $w_{\mu}$ (see Sec. 3) centered at the rational surface $r_{\mu}$ defined by $m-n q\left(r_{\mu}\right)=0$. Let us now add a second mode $b_{\mu}$, of amplitude comparable to the first and with helicity $\mu^{\prime}$ incommensurate with $\mu: \mathrm{m} / \mathrm{n} \neq \mathrm{m}^{\prime} / \mathrm{n}^{\prime}$. Aspects of this complicated 
situation can be characterized by the important stochasticity parameter S (Chirikov 1969; Zaslavskii \& Chirikov 1971), defined for this two-mode example by the ratio of the typical island width to the separation of. the island centers:

$$
s \equiv \frac{1}{2}\left(w_{\mu}+w_{\mu}\right) /\left(r_{\mu}-r_{\mu}\right)
$$

Even if the islands are well separated $(S<1)$, the separatrix of each island is now destroyed locally, in a thin layer centered around the original separatrix. The width of this stochastic layer can be estimated (Chirikov 1969; Rechester \& Stix 1976; Stix 1976) to be of order $\varepsilon^{-3} \exp (-\pi / 2 \varepsilon)$, where $\varepsilon \simeq \mathrm{S} / 4$. Within the stochastic layer, the magnetic lines wander randomly-- that is, in a volume rather than on a surface. $f$ For extremely small perturbations $s \ll 1$, the Kolmogorov-Arnol'd-Moser (KAM) theorem Kolmogorov 1954; Arnol'd 1963; Moser 1973; Ford 1975; Walker \& Ford 1969) assures us that the separatrix destruction is benign. In a measure-theoretic sense, the part of phase space with destroyed surfaces is small and near the original separatrices. Slightly away from the original separatrices, flux surfaces persist with shapes altered only slightly from the unperturbed ones. However, as $S$ increases, the stochastic layers expand, eventually "overlapping" (Chirikov 1969) and filling essentially the entire volume contained within the two original islands. This is the so-called "stochastic instability."

'A clear, non-mathematical introduction to the ergodic problem and various kinds of phase space flows can be found in Balescu's book (1975, pp. 695-729). 
For more complex situations involving many islands, one defines $S$ as in Eq. (2) except that one uses a mean width $w$ and mean separation $\Delta$ between adjacent resonances: $S \stackrel{i}{\simeq} w / \Delta$. Nominally, the transition to extensive stochasticity occurs at island overlap: $\quad S \simeq 1$. (In practice, substantial stochasticity occurs at values of ${ }^{\prime} \mathrm{s}$ somewhat smaller than one, corresponding to overlap of secondary islands arising from high order resonances.) Near the stochasticity threshold $S \leqslant 1$ the global topology of the lines is complicated. The phase space is divided, including both ergodic and trapping (island) regions with roughly equal measure. We shall assume that our system is well into the stochastic regime, $S>1$, in which case the measure of the trapping component is assumed to be very small, though finite. The analýtic theory which we develop neglects the small trapping component. For our purposes, this approximation has been adequately justified by many numerical experiments (Chirikov 1977; Smith \& Kaufman 1975; Smith \& Kaufman 1976; Smith 1977 a \& b; Smith \& Kaufman 1978; Ford 1975; Walker \& Ford 1969).

A practical measure of the stochasticity is the magnetic diffusion coefficient $D_{m}$, which relates the radial dispersion $\left\langle\left(r-r_{0}\right)^{2}\right\rangle \equiv\left\langle\delta r^{2}\right\rangle$ of the lines to toroidal distance $\delta z=R\left(\phi-\phi_{0}\right)$ (essentially the distance along an unperturbed line):

$$
D_{m}=\lim _{\delta z \rightarrow \infty}\left\langle\delta r^{2}\right\rangle / 2 \delta z
$$


The average here is over an ensemble, each realization of which is defined by a line which intersects a given unperturbed surface $r=r_{0}$ (constant over the ensemble) at a given poloidal and toroidal angle (distributed uniformly in $[0,2 \pi$ ) over the ensemb1e).

More detalled information about the stochastic field can be obtained from the correlation function $c \equiv\langle b b\rangle$ of the radial perturbation. We will need both the Eulerian form

$$
c\left(r, \theta, z, t ; r^{\prime}, \theta^{\prime}, z^{\prime}, t^{\prime}\right) \equiv\left\langle b(r, \theta, z, t) b\left(r^{\prime}, \theta^{\prime}, z^{\prime}, t^{\prime}\right)\right\rangle
$$

and the Lagrangian form

$$
C_{L}\left(z, t ; z^{\prime}, t^{\prime}\right) \equiv C\left[r(z), \theta(z), z, t ; r\left(z^{\prime}\right), \theta\left(z^{\prime}\right), z^{\prime}, t^{\prime}\right] .
$$

It is the equal time Lagrangian function $C_{L}\left(z ; z^{\prime}\right)$ which determines the magnetic diffusion coefficient:

$$
D_{m}=\lim _{\delta z_{\rightarrow \infty}}\left(\frac{1}{2 \delta z} \int \mathrm{dz}_{0} \int_{z_{0}}^{z_{0}+\delta z} \mathrm{dz} \int_{z_{0}}^{z_{0}+\delta z} d z^{\prime \prime} c_{L}\left(z^{\prime} ; z^{\prime \prime} \mid z_{o}\right) p\left(z_{0}\right)\right)
$$

Here $C_{L}\left(z^{\prime} ; z^{\prime \prime} \mid z_{0}\right)$ is the conditional probability for measurements at $z^{\prime}, z^{\prime \prime}$, given the initial condition of $z_{0}$, and $p\left(z_{0}\right)$ represents a uniform distribution of initial conditions in the toroidal direction. Notice that because our initial ensemble is singular in radius, i.e.,

$$
\left\langle\delta\left[x-r\left(z_{0}\right)\right] \delta\left[\theta-\theta\left(z_{0}\right)\right] \delta\left(z-z_{o}\right)\right\rangle=\delta\left(x-r_{0}\right)(2 \pi)^{-1}(2 \pi R)^{-1},
$$


the Lagrangian function is not stationary in general, $C_{L}\left(z ; z^{\prime}\right)$ $\neq \mathrm{C}_{\mathrm{I}}\left(\mathrm{z}-\mathrm{z}^{\prime}\right)$, though it does become so asymptotically, for $r\left(z^{\prime}\right)$ greater than a radial correlation length. If we ignore the radial dependence of $C$, an approximation we can justify in certain interesting limits (see Sec. 4), then ${ }^{\prime} C_{L}$ is truly stationary and

$$
D_{m}=\int_{U}^{\infty} d z C_{L}(z)
$$

Because it is in general the Eulerian correlation function which is readily computable or measurable, evaluation of this formula involving the Lagrangian function presents certain ${ }^{\vdots}$ technical difficulties.

The $\mathrm{z}$ dependence of $C_{L}$ contains at least two physically distinct contributions. We can write qualitatively

$$
C_{L}(z) \sim C(0) C_{\|}\left(z ; I_{0}\right) \exp \left(-z / L_{K}\right)
$$

where $C(0)$ measures the intensity of the fluctuations; $C_{\|}$is an Eulerian correlation function, of unit area and width $I_{0}$ in $\mathbf{z}$, describing loss of magnetic phase correlations àlong an unperturbed line (an effect present already in linear theory); and the exponential factor describes the intrinsically nonlinear loss of correlations due to the mixing property [in the technical sense (Zaslavskii \& Chirikov 1971)] of the stochastic system of lines. The quantities $L_{O}$ and $L_{K}$ are discussed in more detail in sec. 3 . In the conventional limit of a closely spaced or almost continuous 
spectrum (sometimes inadequately called the "quasilinear" limit), we have by definition $\mathrm{L}_{\mathrm{O}} \ll \mathrm{L}_{\mathrm{K}}$; so

$$
D_{m} \simeq D_{m}^{(0)} \equiv C(0) \int_{0}^{\infty} d z C_{\|}^{\left(z ; L_{0}\right)}
$$

Evaluation of this integral is implicit in the particle calculations of Sec. 4; the result is

$$
\left.\mathrm{D}_{\mathrm{m}}^{(0)}=\pi R<\left|\delta B_{\mathrm{r}} / \mathrm{B}\right|^{2}\right\rangle
$$

Aspects of this calculation have been discussed by Rosenbluth, Sagdeev, Taylor \& Zaslavskii (1966) who huwever do not discuss the nonlinear mixing and effectively take $L_{K}=\infty$. That $L_{K}$ is finite is crucial for the particle transport problem of present concern, as we will see.

In the opposite limit $\mathrm{L}_{\mathrm{K}}<\mathrm{L}_{\mathrm{O}}$, it is easy to estimate that

$$
D_{m} \sim D_{m}^{(0)}\left(L_{K} / L_{0}\right)<D_{m}^{(0)}
$$

Because in general $L_{K}$ is a function of $D_{m}$, Eq. (5) is a selfconsistent equation to be solved for $D_{m}$.

The main point of this paper is that radial diffusion of magnetic lines can cause radial particle transport rapid compared to the conventional collisional cross-field transport across well-formed surfaces. ${ }^{\dagger}$

${ }^{\dagger}$ A comprehensive review of neoclassical transport is given by Hinton and Hazeltine (1976). We are aware of no comparable review of anomalous transport due to turbulent fluctuations. 
In the small gyroradius approximation, to which we restrict ourselves throughout this paper, the radial velocity of a particle is dominantly the radial projection of its parallel velocity:

$$
v_{r}=v_{\|} b
$$

(There is also another small contribution to $v_{r}$, due to collisional diffusion, which will be important later.) The timedependent radial particle dispersion

$$
\left\langle\delta r^{2}(t)\right\rangle=2 \int_{0}^{t} d \tau(t-\tau)\left\langle V_{\| !}(\tau) b(\tau) V_{\|}(0) b(0)\right\rangle
$$

(we assume stationarity for simplicity) defines by differentiation a time-dependent function

$$
D(t) \equiv \frac{1}{2} \frac{d}{d t}\left\langle\delta x^{2}(t)\right\rangle=\int_{0}^{t} d \tau\left\langle V_{\|}(\tau) b(\tau) v_{\|}(0) b(0)\right\rangle
$$

which becomes asymptotically a true diffusion coefficient

$$
D=\lim _{t \rightarrow \infty} D(t)
$$

if the limit exists and is nonvanishing. Because parallel transport rates are rapid, $D$ can be large. In addition to the usual complication of a Lagrangian function entering into Eq. (7), an additional problem arises here from the four-point nature of that function. This, of course, reflects the explicit presence of two 
physically distinct random processes: radial line diffusion and parallel particle transport. Also implicit in the $\tau$ dependence of $\langle\mathrm{VbVb}\rangle$ are the effects of transport across the lines.

Formula (7) can be simplified in the completely collisionless, streaming limit (of little physical interest for transport in tokamaks). Since we have ignored gradients of $|\underset{\sim}{B}|$, the parallel velocity is constant in each realization

$$
D=\left\langle v^{2} \int_{0}^{\infty} d \tau\langle b(\tau) b(0)\rangle\right\rangle_{T}
$$

where $<{ }_{T}$ denotes an average over a thermal velocity distribution. If the magnetic fleld does not fluctuate in time, then

$$
\mathrm{D} \simeq \overline{\mathrm{V}} \int_{0}^{\infty} \dot{\mathrm{dz}} \mathrm{C}_{\mathrm{L}}(\mathrm{z})=\overline{\mathrm{v}} \mathrm{D}_{\mathrm{m}},
$$

where $\overline{\mathrm{V}} \equiv\left\langle\mathrm{v}^{2}\right\rangle_{\mathrm{T}}^{1 / 2}$ and $\mathrm{dz} \equiv \overline{\mathrm{V}} \mathrm{d} \tau$, and where we have ignored the distinction between the increment of length ds along a line and the toroidal increment $\mathrm{Rd} \phi$. The result (8) has been known for some time (Jokipii \& Parker 1969) and reduces the problem to the computation of the magnetic diffusion coefficient.

In the case of more physical relevance when particles suffer many collisions before diffusing from the device, the four-point function in Eq. (7) cannot be factored. It is convenient, however, to write $D(t)=\bar{v} D_{m} d(t)$ for some function $d(t)$. Sections 4 and 5 are devoted to finding $d(t)$ and to proving that the asymptotic diffusion coefficient $D$ takes the form 


$$
D=\bar{V} D_{m} d\left(\tau_{c}\right)
$$

where $\tau_{c}$ is the correlation time for particle memory of the line on which it resided at $t=0$. This time, which has also to be computed, is in general the shortest of the three times $\left\{\tau_{b}, \tau_{\delta}^{(\theta)}, \tau_{\delta}^{(r)}\right\}$, where $\tau_{b}$ is the temporal correlation time of the background turbulence and $\tau_{\delta}^{(\theta)}, \tau_{\delta}^{(r)}$ are the times for cross-field collisional diffusion over an azimuthal or radial correlation length of the field. The function $d(t)$ describes the physics of parallel transport, when particles are constrained to remain indefinitely on their initial lines. In $F i g$. I we sketch $d(t)$ for a case of small $L_{o}$. We can then identify three physically distinct regimes.

(1) $0<t<t_{0} \equiv \mathrm{L}_{\mathrm{Q}} / \overline{\mathrm{V}}$ : the lines move freely, $\delta \mathrm{r}_{;}^{2} \sim \mathrm{z}^{2} \sim \overline{\mathrm{v}}^{2} \mathrm{t}^{2}$;

(2) $t_{0}<t<v^{-1}$ : the lines diffuse, $\delta r^{2} \sim D_{1 H} z$, but particles move freely along the lines, $z=\bar{v} t$;

(3) $v^{-1}<t$ : a "double diffusion" limit there the parallel position of a particle is a collisional random walk, so that $\delta r^{2} \sim D_{m}\left(D_{\eta} t\right)^{1 / 2}$, or with $q_{\mid} \sim \bar{v}^{2} / \nu, d(t) \sim(\nu t)^{-1 / 2}$, $v t \gg 1$.

Perpendicular transport moves particles across linès, which mix exponentially rapidly because of the stochasticity: One finds that approximately

${ }^{T}$ The double diffusion effect was first pointed out to one of us (JAK) by M.N. Rosenbluth (private communication, 1977). See also Rechester \& Rosenbluth 1.978. 


$$
L_{\delta} \equiv z\left(\tau_{\delta}\right)=L_{K} \ln \left[\left(\frac{\bar{\tau}_{\perp}}{\tau_{\delta}}\right)^{1 / 2}\right]
$$

where

$$
z(\tau)= \begin{cases}\bar{v} \tau, & v \tau<1 ; \\ \left(2 \mathrm{D}_{\|} \tau\right)^{1 / 2}, & \nu \tau>1 ; ;\end{cases}
$$

and where

$$
\bar{\tau}_{\perp} \equiv\left(\bar{k}^{2} D_{\perp}\right)^{-1}
$$

is the time to diffuse a typical perpendicular wavelength $\overrightarrow{\mathrm{k}}^{-1}$. Here the perpendicular diffusion coefficient $D_{\perp}$ arise from either classical. or turbulent collisions. If $\ell$ is the mean free path $\ell \equiv \bar{V} / \nu$ and $\tau_{\delta}<\tau_{b}$, one then finds from Eqs. (9) and (10) that

$$
D=D_{m} \begin{cases}\bar{V}, & \ell / L_{\delta}>1 ; \\ D_{\|} / L_{\delta}, & \ell / L_{\delta}<1 ; ;\end{cases}
$$

where Eqs. (1la,b) define respectively the collisionless and collisional regimes. Notice that although $t_{\delta}$ depends only logarithmically on the perpendicular collisional transport, if $\mathrm{D}_{1}$ were neglected completely the asymptotic diffusion coefficient: would vanish because of the slow, double diffusion law for purely paraliel transport.

The result (11) agrees with the heuristic arguments of $R R$. It is, however, subject to the restrictions 
(a) $\mathrm{L}_{\mathrm{O}} / \mathrm{L}_{\mathrm{K}}<1$,
(b) $\mathrm{L}_{\mathrm{O}} / l<1$,
(c) $\mathrm{D}_{1}<\mathrm{D}$,

(d) $\tau_{\delta}<\tau_{b}$ (for $\left.\ell / L_{\delta}<1\right)$

The ordering in Eq. (12a) is of particular interest because, as we will. see, it holds for moderate values of stochasticity, not too far above threshnld, and would be passed thiuuyl flrse ás unstable fluctuations grew from noise. However, other limits are certainly possible, and can be physically realized. Restriction ( $12 \mathrm{~b}$ ) is relaxed in Sec. 4. Restrictions $(12 \mathrm{a}, \mathrm{c}, \mathrm{d})$ are relaxed qualitatively in sec. 6. A detailed discussion of the more general cases will be given elsewhere.

This completes our qualitative discussion. Detailed justification of these results and quantitative computation of numerical coefficients require a nontrivial kinetic theory, which we develop in the remainder of the paper. In Sec. 3 we define and diecuss the characteristic parameters which arise naturally. In sec. 4 we give a heuristic derivation of the four-point Iagrangian correlation function. First, we study the case of purely parallel transport, $D_{\perp}=0$, and compute $d(t)$. We show that the double diffusion law can be understood in terms of a peculiar negative tail of the radial velocity correlation function, which arises for times $t>v^{-1}$ because of collisional diffusion of particles, with average position the mean free path, backwards into the 
regime of significant Lagrangian correlations $\left(z \leqslant L_{0}\right)$ of the field lines. Next, we consider the effects of $D_{\perp}$. Because we assume $\mathrm{D}_{1} \ll \mathrm{D}$, we can introduce the perpendicular transport process as a perturbation. We then derive differential moment equations describing the perpendicular motion of particles relative to a given line and use these to find the results in Eqs. $(9,11)$ with, for the first time, a specific formula for the mixing length $L_{k}$. We find that the moment equations are formally identical to some of Dupree's equations (1972) for the relative motion of particles in small regions of Vlasov phase space. This identity is not accidental but rather profound, and we comment on its significance.

In Sec. 5 we formulate and, to some extent, solve the rigorous kinetic theory. We describe the random motion of the test electron by a stochastic Liouville equation. To find an equation for the averaged particle motion, we find it convenient and natural to introduce the joint probability distribution $\mathrm{K}$ for the particles and field. Because of the resonances in the $\mathrm{K}$ equation which are in fact responsible for the stochasticity, a proper renormalization is essential. However, we find that it is inadequate and qualitatively incorrect to employ the conventional renormalized quasilinear theory (QLT), which factors $\mathrm{K}$ into the product of the singlet distributions for the particles and field separately. Rather, to correctly treat the diffusion of particles relative to lines, one must retain field-particle correlations. To do this, we consider a limit of moderate stochasticity, in which the radial increment of a line integrated though one resonance is small, and reduce the formally exact equation for $\mathrm{K}$ to $\mathrm{a}$ 
diffusion equation for the motion of the particle relative to its initial line. Moments derived from this equation agree with those computed heuristically in Sec. 4. Again, this kinetic equation is closely related to the equations Dupree uses in his discussion of phase space granulation (persistence of two-particle correlations, or "clumping") in vlasov turbulence. ' In retrospect, it is not surprising that the two problems are logically related, since both involve the complicated phase space topolngy nf a stochastic instability. However, the detailed relation of Dupree's work to stochasticity theory has remained somewhat obscure. Because we have succeeded in merging intuitive stochasticity notions with a detailed kinetic theory, and because our renormalization techniques are more systematic than Dupree's, we hope that our results, will provide useful insight and procedures for a variety of plasma turbulence problems.

\section{Characteristic Parameters}

In this section we discuss the characteristic parameters of the theory. For convenience, these are presented in a reference format, with numbered key phrases followed by the symbols of the most important quantities introduced in that subsection. We begin with those parameters defined even in the absence of stochasticity of the lines. 


\subsection{Shear $\left(\mathrm{L}_{\mathrm{s}}\right)$.}

Shear of the unperturbed lines $\left(B_{r}=0\right)$ is essential for at least two reasons. First, conventional islands have finite width only for non-zero shear. Second, properties of the background fluctuations depend on shear. The shear length $\mathrm{L}_{s}$ is defined as

$$
\mathrm{L}_{\mathrm{s}} \equiv \mathrm{Rq} / \mathrm{s},
$$

where the shear parameter $\mathbf{s}$ is defined as

$$
s \equiv d(\ln q) / d(\ln r)
$$

In the present work, these quantities are to be evaluated at a reference radius $r=r_{0}$. Typically $s \simeq 1$, the ordering which we assume throughout. For current profiles typical of normal tokamak discharges, which decrease monotonically from the center to zero at the wall, $+0 \leq s \leq 2$.

\subsection{Degree of Radial Localization $\left(\Delta r, L_{0}, \Delta k_{\| l}\right)$}

Finite shear implies that the radial eigenfunctions of the background fluctuations are not plane waves but are rather functions localized in radius. Let us define the parallel wavenumber $\mathrm{k}_{\|}$as

We are indebted to G. Rewoldt for emphasizing this point (private communication, 1977). 


$$
\mathrm{k}_{\|} \equiv \mathrm{R}^{-1}(\mathrm{~m} / \mathrm{q}-\mathrm{n})
$$

Then, if the fluctuations are localized to a region $\pm \delta \dot{r} \equiv \pm \Delta r / 2$ around a rational surface $k_{\|}=0$, the spread in parallel wavenumber $\Delta \mathrm{k}_{\|} \equiv 2\left|\delta \mathrm{k}_{\|}\right|$is determined from

$$
\delta k_{\|}=-\frac{\mathrm{m}}{\mathrm{Rq}^{2}} \frac{\mathrm{dq}}{\mathrm{dr}} \delta \mathrm{r}=-\mathrm{L}_{\mathrm{s}}^{-1}\left(\mathrm{k}_{\theta} \delta \mathrm{r}\right) \sim \mathrm{L}_{\mathrm{s}}^{-1}
$$

In writing the last approximation, we assumed that the azimuthal and radial extents of the modes are comparable, as is suggested by recent microinstability calculations (Chen, Rutherford \& Tang 1977; Rechester and Rosenbluth 1978). The parallel length associated with the localization is defined to be $\mathrm{L}_{0}$ :

$$
\mathrm{L}_{\mathrm{O}} \equiv 2 \pi /\left|\delta \mathrm{k}_{\|}\right| \sim \mathrm{L}_{\mathrm{S}}
$$

'lhere is a distinction between the radial localization length $\delta r$, a property of single eigenfunctions, and the radial correlation length $\rho_{c}$, a statistical quantity. In general, perturbed quantities possess expansions of the form

$$
\begin{aligned}
\delta B(\underset{\mu}{x}, t) & =\sum_{\mu} b_{\mu l}(r, t) \exp [i(m \theta-n \phi)] \\
b_{\mu}(r, t) & =\sum_{\ell} b_{\mu \ell}(t) H_{\ell}\left(r-r_{\mu}\right),
\end{aligned}
$$

where $\mathrm{H}_{\ell}$ is a radial eigenfunction centered around the rational surface $m-n q\left(r_{\mu}\right)=0$. Because of rotational invariance, the 
two-point correlation function can be written as

$$
\langle\delta B(\underset{\sim}{x}+\underset{\sim}{\rho}) \delta B(\underset{\sim}{x})\rangle=\sum_{\mu}\left\langle b_{\mu}(r+\rho) b_{-\mu}(r)\right\rangle \exp [i(m \delta \theta-n \delta \phi)],
$$

where

$$
\left\langle b_{\mu}(r+\rho) b_{-\mu}(r)\right\rangle=\sum_{\ell, \ell^{\prime}}\left\langle b_{\mu, \ell} b_{-\mu, \ell^{i}}>\mathrm{H}_{\ell}\left(r+\rho-r_{\mu}\right) H_{\ell},\left(r-r_{\mu}\right) .\right.
$$

The characteristic scale of variation of the intensity $\left\langle\left|b_{\mu}(r)\right|^{2}\right\rangle$ is defined to be $\delta \mathrm{r}$. The correlation length $\rho_{\mathrm{C}}$ describes the variation of Eq. (14) with $\rho$; it is comparable to or less than $\delta r$

\subsection{Stochasticity Parameter $\left(S, w_{\mu}\right)$}

The total wavenumber spread $\Delta \mathrm{k} \|$ determines the number of resonances $\mathrm{N}$ which contribute at any given radius $r_{0}$ :

$$
\dot{\mathrm{N}}=\sum_{\mu}=\sum_{\mathrm{In}, \mathrm{k}_{\|}} \simeq \sum_{\mathrm{m}} \mathrm{R} \int \mathrm{dk_{ \| }}=\Delta \mathrm{mR} \Delta \mathrm{k}_{\|},
$$

where $\Delta \mathrm{m} \sim \mathrm{m}$ is the number of excited poloidal harmonics. The mean separation $\Delta \equiv \Delta r / N$ between these resonances then follows from Eqs. (15) and (13):

$$
\Delta=\left(\frac{q}{s}\right) \frac{1}{\left(\Delta m k_{\theta}\right)}
$$

The stochasticity parameter $s$ was defined in Eq. (2). If $\mathrm{b}_{\mu}$ is even about $r=r_{0}$, then the island width $w_{\mu}$ is 


$$
\mathrm{w}_{\mu}=4\left(2 \mathrm{~L}_{\mathrm{S}} \mathrm{b}_{\mu} / \mathrm{k}_{\theta}\right)^{1 / 2}
$$

For drift fluctuations, one expects the radial dependence of $b_{\mu}$ to be odd about $r_{\mu}$ (Callen 1977; Chen, Rutherford \& Tang 1977). However, the precise form of $w_{\mu}$ is unimportant since our theory assumes $\mathrm{S} \gg 1$, in which case individual island structures are destroyed. As a practical matter, substantial stochasticity usually means $S>1$, not, for example, $S>10^{3}$.

\subsection{Collisions $\left(\nu, l, D_{\|}, D_{\perp}\right)$}

Parallel transport is described by the collision frequency $\nu$, the thermal mean free path $\ell \equiv \bar{V} / \nu$, and the parallel diffusion coefficient $\mathrm{D} \equiv \ell^{2} v=\overline{\mathrm{V}}^{2} / \nu$. We do not specify $v$, which can include the effects of turbulence. However, we reguire that the scales of fluctuations which cause the collisions are small compared to the characteristic scales for stochastic transport. Using the kinetic techniques of sec. 5, we could remove this restriction at the expense of increased analytical complexity. A prototype calculation can be found in Krommes \& Oberman (1976).

It is convenient to assume that the effective (turbulent) collision frequency for cross-field transport is comparable to or greater than $v$ but that the associated step lengths for perpendicular random walk are effectively zero. This is a white noise approximation in which perpendicular transport is described solely by a diffusion coefficient $D_{\perp}$. This approximation may not be well justified for some kinas of low-frequency turbulence. 
However, one expects that the influence of $D_{\perp}$ on the stochastic transport remains qualitatively unchanged provided that the scales involved do not exceed those for the stochastic random walk.

We always assume that $D_{\perp}$ is small: $D_{\perp} \ll D$.

\subsection{Exponentiation Length $\left(\mathrm{L}_{K}\right)$}

An important parameter of stochastic fields is the exponentiation length $\mathrm{L}_{\mathrm{K}}$. This is defined as the distance $\mathrm{z}$ required for two typical adjacent lines to diverge significantly (exponentially) from each other. Thus, the distance $\Delta(z)$ between lines, given initial separation $\Delta_{0}$ at $z=0$, obeys in the simplest cases

$$
\Delta(z)=\Delta_{0} \exp \left(z / L_{K}\right)
$$

The inverse of $L_{K}$ is proportional to the so-called $K$-entropy, introduced by Kulmogoroff (1954) as a measure of the ergodicity of a dynamical system. The exponential character of the divergence is the essence of stochastic instability; it should be contrasted with the case of well-defined flux surfaces where the deviation is linear.

More precisely, $\mathrm{L}_{\mathrm{K}}$ is in general a local function of space and describes differential divergence. Of more interest are global properties of the flux. (We use "flux" in the technical sense of Chirikov (1969), namely to describe evolution parametrized 
by a continuous parameter $z$ as opposed to a "mapping" defined only at discrete points $\mathrm{n}_{\cdot}$ ) An averaged $\mathrm{L}_{K}$ can be defined from the second moment of a typical cartesian component of the vector separation between lines. For example, if $y=R \theta$ one expects that:

$$
\left\langle\Delta y^{2}(z)>\sim \exp \left(2 z / L_{\mathrm{K}^{\prime}}\right)\right.
$$

A related procedure follows by noting that the exponentiation leads to irreversible mixing which, in turn, results in decay to zero of the two-point Lagrangian correlation function. This point is illustrated by a model mapping which Chirikov uses to demonstrate many of the stochasticity concepts with a minimum of algebraic complexity. Chirikov (196:9) considers the mapping for the phase function $\psi$ :

$$
\Psi_{n+1}=K \Psi_{n}
$$

$(K \gg>1)$. We' have

$$
\Psi_{\mathrm{n}}=\mathrm{K}^{\mathrm{n}} \Psi_{0}
$$

so that

$$
\begin{gathered}
C(n) \equiv\left\langle\exp \left(i \Psi_{n}\right) \exp \left(-i \Psi_{0}\right)>=\int_{0}^{2 \pi} \frac{d \Psi_{0}}{2 \pi} \exp \left[i\left(K^{n}-1\right) \Psi_{0}\right],\right. \\
|C(n)| \simeq \exp (-n \ln K): / 2 \pi
\end{gathered}
$$


for $\mathrm{K}$ not an integer and $\mathrm{n}>0$. On the other hand, the growth of small phase perturbations iterated through the mapping obeys

$$
\begin{aligned}
\Delta \Psi_{\mathrm{n}+1} & =\mathrm{K} \Delta \Psi_{\mathrm{n}}, \\
\Delta \Psi_{\mathrm{n}} & =\exp (\mathrm{n} \ln \mathrm{K}) \Psi_{\mathrm{o}},
\end{aligned}
$$

so that perturbations grow exponentially at the same rate at which correlations decay.

In the present problem we find that for $\mathrm{L}_{\mathrm{O}} / \mathrm{L}_{\mathrm{K}}<1$,

$$
L_{K}=L_{s}\left(\frac{1}{2} \bar{k}_{\theta}^{2} D_{m}^{\prime \prime} L_{s}\right)^{-1 / 3},
$$

where $D_{m}^{\prime \prime} \simeq D_{m}$. This length has scaling identical to the convential "resonance broadening" correlation length $\mathrm{L}_{\mathrm{C}}$ of renormalized QLT (Dupree 1966). There is, however, an important physical distinction between $L_{K}$ and $L_{C}$ which we now discuss.

\subsection{Eulerian and Lagrangian Correlation Lengths $\left(\mathrm{L}_{C}, \mathrm{~L}_{\mathrm{O}}, \overline{\mathrm{k}}_{\theta}, \mathrm{L}_{K}\right)$}

The Lagrangian correlation function of the field taken along an unperturbed line can be computed rigorously from $C_{L}\left(z ; z^{\prime}\right) \equiv \int d r d y d r^{\prime} d y^{\prime}\left\langle b(r, y, z) \tilde{P}(r, y, z) \tilde{P}\left(r^{\prime}, y^{\prime}, z^{\prime}\right) b\left(r^{\prime}, y^{\prime}, z^{\prime}\right)\right\rangle$,

where the random Liouville function $\tilde{P}(r, y, z) \equiv \delta[r-r(z)] \delta[(y-y(z)]$ 
for the lines obeys

$$
\frac{\partial}{\partial z} \tilde{p}+\frac{\left(r-r^{\prime}\right)}{I_{s}} \frac{\partial}{\partial y} \tilde{P}+b \frac{\partial}{\partial r} \tilde{P}=0 .
$$

In writing the second term of Eq. (18), we have assumed that the radial step size for the stochastic random walk is smail compared to the scale of variation of $q$ (the equilibrium scale length). This is easily verified a posteriori. For distances $z-z^{\prime}$ sufficiently large, it is plausible that Eq., (17) can be factored according to

$C_{L}\left(z ; z^{\prime}\right) \simeq \int d r d y d r^{\prime} d y^{\prime} R\left(r, y, z ; r^{\prime}, y^{\prime}, z^{\prime}\right) C_{E}\left(r, y, z ; r^{\prime}, y^{\prime}, z^{\prime}\right)$,

where $C_{E}$ is the Eulerian correlation function $\langle b b\rangle$ for the field strength and $R$ is the averaged Green's function for a line passing through $\left(x^{\prime}, y^{\prime}, z^{\prime}\right)$. To compute $C_{E}$ we can average over $\left(y^{\prime}, z^{\prime}\right)$ holding $\delta y \equiv y-y^{\prime}, \delta z$ fixed:

$$
\begin{aligned}
C_{E}\left(r, \delta y, \delta z ; r^{\prime}\right)= & \int \frac{d \theta_{o}}{2 \pi} \frac{d \phi_{0}}{2 \pi} \sum_{\mu, \mu^{\prime}}\left\langle b_{\mu}(r) b_{\mu}\left(r^{\prime}\right)\right\rangle \\
& \times \exp \left\{i(m \delta \theta-n \delta \phi)+\left[i\left(m+m^{\prime}\right) \theta_{0}-\left(n+n^{\prime}\right) \phi_{0}\right]\right\} \\
= & \sum_{\mu} F_{\mu}\left(r ; r^{\prime}\right) \exp \left(i k_{\theta} \delta y+i k_{\|} \delta z\right)
\end{aligned}
$$

where $F_{\mu}\left(r, r^{\prime}\right) \equiv<b_{\mu}(r) b_{-\mu}\left(r^{\prime}\right)>$. If $F_{\mu}$ is separable in $m$ and $k_{\|}$, as would be the case if all harmonics were excited equally in some band, and if we ignore the $r$ dependence, then

$$
C_{E}(\delta y, \delta z) \simeq C_{\perp}(\delta y) c_{\|}(\delta z)
$$


The $z$ variation of $C_{\|}$defines the Eulerian parallel correlation length, of the order of $\mathrm{L}_{0}$. The $y$ variation of $\mathrm{C}_{\perp}$ gives the decay of azimuthal correlations, on a scale $\overline{\mathrm{k}}_{\theta}^{-1}$, where $\overline{\mathrm{k}}_{\theta}$ is a typical azimuthal wavenumber.

For $|\delta z|>L_{0}, R$ will obey a diffusion equation. For $\delta z \geq 0$ this is

$$
\left(\frac{\partial}{\partial z}+\frac{\left(r-r^{\prime}\right)}{L_{s}} \frac{\partial}{\partial y}-D_{m} \cdot \frac{\partial^{2}}{\partial r^{2}}\right) R\left(r, y, z ; r^{\prime}, y^{\prime}, z^{\prime}\right)=H\left(z-z^{\prime}\right) \delta(\delta y) \delta(\delta z),
$$

where $D_{m}$ is the diffusion coefficient of the lines. If we measure all lengths in terms of $\mathrm{L}_{S}$, the solution of Eq. (20) is

$$
R(\delta r, \delta y, \delta z)=H(\delta z) P_{r}(\delta r, \delta z) P_{y}\left(\delta y-\frac{1}{2} \delta r \delta z, \delta z\right)
$$

where

$$
P_{r}(r, z) \equiv(4 \pi D z)^{-1 / 2} \exp \left(-r^{2} / 4 D z\right)
$$

describes radial diffusion of the lines and

$$
P_{y}(y, z)=\left(\frac{1}{3} \pi D z^{3}\right)^{-1 / 2} \exp \left[-y^{2} /\left(\frac{1}{3} D z^{3}\right)\right]
$$

describes the associated azimuthal motion. Now the probability of azimuthal position $y$,

$$
\int d(\delta r) R=\left(\frac{4 \pi}{3} D z^{3}\right)^{-1 / 2} \exp \left[-y^{2} /\left(\frac{4}{3} D z^{3}\right)\right]
$$

has as Fourier transform $\exp \left(-\frac{1}{2} \mathrm{k}_{\theta}^{2}\left\langle\mathrm{y}^{2}\right\rangle\right)$, where $\left\langle\mathrm{y}^{2}\right\rangle=(2 / 3) \mathrm{Dz}{ }^{3}$. 
The correlation length for the $k_{\theta}$ component is defined to be $\mathrm{L}_{C}:$

$$
L_{c} \equiv L_{s}\left(\frac{1}{3} k_{\theta}^{2} D_{m} L_{s}\right)=1 / 3
$$

This lengthi is infinite for systems with zero shear. Similarly, Fourier decomposition of the radial probability yields the radial correlation length $L_{C}{ }_{C}^{(r)}=\left(k_{r}^{2} D_{m}\right)^{-1}$, which remains finite for zero shear.

The factorization (19) is the "well-known Direct Interaction Approximation (DIA) (Kraichnan 1961; 1958). For the present, non-self-consistent problem, the DIA is identical to renormalized QLT. It is, apparently, not widely understood that this theory is valid only for $\delta z \gg L_{C}$. Indeed, for $\delta z<L_{C} \cdot L_{K}$ pairs of Iines exponentiate from each other, so the body of: the Lagrangian function has an envelope more like: $\exp \left[-\frac{1}{2} \mathrm{k}_{\theta}^{2}<\mathrm{y}^{2}(\delta z)>\right]$, with $\left\langle y^{2}\right\rangle \sim \exp \left(2 \delta z / I_{K}\right)$. Only when lines diffuse independently, for $\delta z>L_{K}$ or atypically short wavelengths, is the envelope behavior $\exp \left[-\left(\delta z / L_{C}\right)^{3}\right]$ which follows from $E_{\text {: }}$. (19) appropriate. Depending on the strength and spectral details of the fluctuations, $\mathrm{L}_{\mathrm{K}}$ can be either larger or smaller than $\mathrm{L}_{\mathrm{o}}$. The phrase "almost continuous spectrum" is defined to mean $\mathrm{L}_{\mathrm{O}} \ll \mathrm{L}_{\mathrm{K}}$. This is a conventional limit, appropriate for fluctuation amplitudes not too far above the stochasticity threshold. $t$

†Private conversations with RR (1977) have convinced us that this is the limit intended by those authors (Rechester \& Rosenbluth 1978), though this is not made clear in their paper. 
In this paper, we shall study only this limit in detail. However, our approximate kinetic equations are valid more generally; we comment briefly on the opposite limit in Sec. 6 .

In general, the actual decay of $C_{L}$ is governed by the smalier of $L_{O}$ and $L_{K}$. If $L_{K}$ were infinite, $C_{L}$ would be a periodic series of spikes, of width $L_{o}$. The decay of a spike is due to nonresonant phase mixing (Tetreault 1976); the periodicity follows from the discrete nature of the spectrum. This periodicity is removed by $\mathrm{L}_{K}$, which describes the loss of correlations due to the intrinsically nonlinear stochastic effects. It is this nonlinear broadening which justifies the usual approximation of integration rather than summation over $k_{\|}$in the formulas defining $C_{L}$. The integration then gives (3), where $C_{\|}$. has (effectively) a single peak around $z=0$ of width $L_{0}$. For the computation of $D_{m}$ with $L_{O} \ll L_{K}$, the slow decay $\exp \left(-z / L_{K}\right)$ can now be ignored. As we will see, this neglect is not permissible for the particle transport problem because particles dorninantly follow the lines, which mix on the $L_{K}$ scale.

4. Heuristic Considerations

\subsection{General Formulas}

Armed with the background of Sec. 3, we can now estimate the particle diffusion coefficient by employing heuristic arguments about the correlations between particles and lines. We do not prove our assertions in this section. such a proof can come 
only from a formal kinetic theory which permits estimates of the sizes of the neglected terms. We formulate such a theory in sec. 5 and show its equivalence, in a certain reasonable approximation, to the present heuristic discussion. It is nontrivial to estimate the neglected terms. We will return to this question in future work.

Though we could proceed more generally, we wish for simplicity to neglect the radial dependence of the Fnurier amplitudoc $b_{\mu}(r, t)$ [see definition (1)]. Tn justify this, we observe that the radial distance a particle diffuses in a step time $\tau_{\delta}$ obeys $\left\langle\delta r^{2}>\sim D_{m} L_{\delta}\right.$. If we approximate $\mathrm{L}_{\delta} \sim \mathrm{L}_{\mathrm{K}} \equiv \mathrm{I}_{\mathrm{K}}^{(\theta)}$ and take the radial wavenumber to obey $\mathrm{k}_{\mathrm{r}} \sim \Delta \mathrm{r}^{-1}$, then we can use Eqs. (4), (i6), and (13b) to estimate

$$
{ }_{\mathrm{r}} \delta \mathrm{r} \sim \mathrm{L}_{\mathrm{O}} / \mathrm{L}_{\mathrm{K}}
$$

where

$$
\begin{aligned}
L_{n} / L_{\mathrm{K}} & \sim\left(k_{\theta} \Delta r\right)^{-1 / 3}(\mathrm{q} / \mathrm{s})^{1 / 3}(\mathrm{R} \delta / \Lambda r)^{2 / 3} \\
& \sim(\mathrm{R} \delta / \Delta r)^{2 / 3} \quad\left(\mathrm{~L}_{\mathrm{O}} / \mathrm{L}_{\mathrm{K}}<1\right) .
\end{aligned}
$$

Thus, for $\mathrm{L}_{\mathrm{O}} / \mathrm{L}_{\mathrm{K}}<1$ the radial dependence should be unimportant. This also justifies the stationarity assumption (6).

By inserting (1) into (7), we find

$D(t)=\int_{0}^{t} d \tau \sum_{\mu, \mu^{\prime}}\left\langle v(\tau) v(0) b_{\mu}(\tau) b_{\mu^{\prime}}(0)\right.$

$\left.x \exp \left\{i[m \delta \theta(\tau)-n \delta \phi(\tau)]+\left[i\left(m+m^{\prime}\right) \theta_{0}-\left(n+n^{\prime}\right) \psi_{0}\right]\right\}\right)$, 
where we have introduced the deviations $\{\delta \theta, \delta \phi\}$ from the initial conditions $\left\{\theta_{0}, \phi_{0}\right\}$. The object $D(t)$ remains a weak function of space $\left(r_{0}\right)$, varying on the equilibrium scale length. If we assume reasonably that the stochasticity assures rotational invariance in angle, we can average Eq. (24) over $\left\{\theta_{0}, \phi_{0}\right\}$ to obtain

$$
D(t)=\int_{0}^{t} d \tau \sum_{\mu}\left\langle b_{\mu}(\tau) b_{-\mu}(0) V(\tau) V(0) \exp \left[i k_{\|} z(\tau)+i k_{\theta} y(\tau)\right]\right\rangle,
$$

where we have written

$$
\begin{aligned}
& z \equiv R \delta \phi \\
& y \equiv r_{0}(\delta \theta-\delta \phi / q)
\end{aligned}
$$

Clearly $z$ describes motions along the unperturbed lines while $y$ describes the corresponding perpendicular motions (at constant $r_{0}$ ).

In the present, non-self-consistent problem, $b_{\mu}(\tau)$ and $V(\tau)$ are rigorously statistically independent. However, it does not follow, as is often assumed, that the orbits $\{\delta \theta(\tau), \delta \phi(\tau)\}$ are independent of $b_{\mu}$. That assumption, which amounts to a Gaussian hypothesis or to renormalized quasilinear theory, is false because of the stochastic instability; description of the exponential deviation of adjacent trajectories requires specific phase information which is absent in a Gaussian approximation. We will, however, write approximately 
$\left.D(t) \simeq \int_{0}^{t} d \tau \sum_{\mu} d \tau<b_{\mu}(\tau) b_{-\mu}(0)\right\rangle\left\langle V(\tau) V(0) \exp \left[i k_{\|} z(\tau) \cdot-\frac{1}{2} k_{\theta}^{2}\left\langle Y^{2}(\tau)>\right]\right\rangle\right.$,

where $\left\langle Y^{2}\right\rangle$ describes the azimuthal dispersion of a pair of particles or, equivalently, the averaged square of the azimuthal deviation of one particle from the actual orbit of another. This is to be contrasted with the prescription used in conventional resonance broadening theory (Dupree 1966), where the dispersion of one particle from its unperturbed orbit is considered. The distinction is important. That our procedure is correct follows physically from the fact that it is two-particle correlations which determine the averaged motion of single particles. Mathematically, it is best understood in terms of the systematic kinetic theory presented in Sec. 5 .

We describe the random parallel motion by the solution $\mathrm{P}(\mathrm{x}, \mathrm{V}, \tau)$ of the Fokker-Planck equation for collisional motion along a (locally straight) line. If we integrate over $k \|$ ' we arrive at

$$
\begin{gathered}
D(t)=2 \pi v_{0} \cdot R \int_{0}^{t} \mathrm{~d} \tau \sum_{m} \delta_{m}^{2} r_{b}(\tau) \int_{-\infty}^{\infty} d V d z P(z, v, \tau) v \cdot \delta\left(z ; \Delta k_{\|}\right) \\
\quad \times \exp \left[-\frac{1}{2} k_{\theta}^{2}<Y^{2}>\right],
\end{gathered}
$$

where the modal interisity $\delta_{m}^{2}$ is defined as

$$
\left.\delta_{\mathrm{m}}^{2} \equiv \mathrm{R} \int \mathrm{dk_{ \mu }}|<| \mathrm{b}_{\mu}\right|^{2}(0)>
$$

the normalized correlation function. $r_{b}(\tau)$ describes the temporal fluctuations of the background, and.where 


$$
\delta(x ; \varepsilon) \equiv \frac{1}{2 \pi} \frac{\sin (\varepsilon x / 2)}{(x / 2)} \sim\left\{\begin{array}{ll}
\varepsilon / 2 \pi, & \varepsilon x \ll \pi \\
\delta(x), & \varepsilon x>\pi
\end{array} .\right.
$$

To arrive at the function $\delta\left(z ; \Delta \mathrm{k}_{\mid}\right)$we assumed for explicitness that $\left\langle\left|\mathrm{b}_{\mu}\right|^{2}\right\rangle$ was flat in $\mathrm{k}_{\|}$for $\left|\mathrm{k}_{\|}\right|<\Delta \mathrm{k}_{j} / 2$, and zero elsewhere. More generally, $\delta\left(\mathrm{z} ; \Delta \mathrm{k} \|^{\prime}\right.$ would be replaced by the parallel Eulerian correlation function.

The integration approximation $\Sigma_{\mathrm{k}_{\|}} \simeq \mathrm{R} \int \mathrm{dk} \|$ is physically reasonable because of the island overlap. To justify it mathematically, we can proceed as follows. From the orbit equations for the lines,

$$
\begin{aligned}
& \frac{d y}{d z}=\frac{r}{L_{s}}, \\
& \frac{d r}{d z}=b,
\end{aligned}
$$

we find the dispersion of a typical line to be

$$
\left\langle y^{2}(z)\right\rangle=L_{s}^{-2} \int_{0}^{z} d z_{1} \int_{0}^{z} d^{\prime} \int_{0}^{z} d z_{2} \int_{0}^{z} d z^{\prime}\left\langle b:\left(z^{\prime}\right) b\left(z^{\prime \prime}\right)\right\rangle
$$

With $\mathrm{C}(\mathrm{z}) \equiv\langle\mathrm{b}(\mathrm{z}) \mathrm{b}(0)\rangle$, this becomes

$$
\frac{1}{2} k_{\theta}^{2}\left\langle y^{2}\right\rangle \simeq \frac{1}{8} k_{\theta}^{2} L_{s}^{-2} c(0) z^{4}, z+0
$$

If we were studying the single particle Green's function, we would require that the increment in $k \|$ be small compared to the width set by Eq. (28): 


$$
\delta k_{\|}=k_{\theta} \Delta / L_{s}<\left(k_{\theta}\left|b_{\mu}\right| / L_{s}\right)^{1 / 2} .
$$

Here $\Delta$ is the typical island separation. With $w$ a typical island width, this reduces to $\Delta<w$, or $1<s$, consistent with the stochastic limit. This argument cannot be applied directly to Eq. (26). However, since $\frac{1}{2} d<y^{2}(z)>/ d z\left[=L_{s}^{-2} z<\delta r^{2}(z)>\right]$ is positive and $\frac{1}{2} \mathrm{k}_{\theta}^{2}\left\langle\mathrm{y}^{2}\right\rangle \sim\left(\mathrm{z} / \mathrm{L}_{\mathrm{c}}\right)^{3}$ which (we will find) is the same scale of variation as $\frac{1}{2} \mathrm{k}_{0}^{2}\left\langle\mathrm{Y}^{2}\right\rangle$, the smoothing will certainly be justified at least for large $z$.

The equation of motion for $Y(\tau)$ follows by changing Eqs. (27) to equivalent orbit equations for the particles according to $d / d z=v^{-1}(\tau) d / d \tau$, then supplementing these with random perpendicular velocities, which represent the effects of collisions or microturbulence:

$$
\begin{aligned}
& \frac{d y}{d \tau}=v \frac{\delta r}{L_{s}}+v_{y}(\tau), \\
& \frac{d \delta r}{d \tau}=v b+v_{r}(\tau),
\end{aligned}
$$

The general solution of this system is complicated because the radial perturbation $b$ is a transcendental function of coordinates. However, for $D_{\perp} \ll D$ and for sufficiently small deviations $\{\hat{y}, \hat{r}\}$ of adjacent lines, we can neglect $v_{x}$ in Eq. (29b) and systematically linearize about the orbit of some actual (not unperturbed) line to find 


$$
\begin{aligned}
& \frac{d \hat{y}}{d \tau}=v \frac{\hat{r}}{\bar{L}_{s}}+v_{\perp}(\tau), \\
& \frac{d \hat{r}}{d \tau}=V \frac{\partial b(y, z)}{\partial y} \hat{y}
\end{aligned}
$$

The homogeneous equations $\left(v_{\perp}=0\right)$ are closely related to the so-called tangent flux one uses to define the exponentiation length for this system:

$$
\begin{aligned}
& \frac{\mathrm{d} \Delta \mathrm{y}}{\mathrm{dz}}=\frac{\Delta \mathrm{r}}{\mathrm{L}_{\mathrm{s}}}, \\
& \frac{\mathrm{d} \Delta \mathrm{r}}{\mathrm{dz}}=\frac{\partial \mathrm{b}}{\partial \mathrm{y}} \Delta \mathrm{y} .
\end{aligned}
$$

Their solution describes the exponential divergence of adjacent lines, or the extension and contraction of small area elements, and is responsible for the $\exp \left(-z / L_{K}\right)$ term in approximation (3). The inhomogeneous part of Eqs. (30) describes the azimuthal motion of particles from line to line because of the perpendicular collisional transport.

For sufficiently short time $\hat{y}$ will be negligible and the solution of the parallel transport problem, $\hat{y}=0$, is relevant. We study this ease in sec. 4.2. In Sec. 4.3 we solve Eqs. (31) and (30) and find the cutoff time ${ }^{\mathrm{T}} \delta \cdot$ 


\subsection{Parallel Transport}

The Fokker-Planck distribution $\mathrm{P}$ is classical (Chandrasekhar 1943) :

$P(z, V, \tau)=\left[2 \pi \sigma_{v}^{2}\left(1-r^{2}\right) 2 \pi \sigma_{z}^{2}\right]^{-1 / 2} \exp \left[-\frac{1}{2\left(1-r^{2}\right)}\left(\frac{\delta z^{2}}{\sigma_{z}^{2}}-\frac{2 r \delta z \delta V}{\sigma_{v} \sigma_{z}}+\frac{\delta V^{2}}{\sigma_{v}^{2}}\right)\right]$, where

$$
\begin{aligned}
& \delta V(\tau)=V-\langle V(\tau)\rangle, \delta z(\tau) \equiv z-\langle z(\tau)\rangle, \\
& \langle V(\tau)\rangle \equiv e^{-\nu \tau} v_{0} \equiv\left\langle V\left(\tau \mid v_{0}\right)\right\rangle, \\
& \sigma_{v}^{2}(\tau) \equiv\left(1-e^{-2 \nu \tau}\right) v_{t}^{2} \\
& \langle z(\tau)\rangle \equiv\left\langle z\left(\tau \mid v_{0}\right)\right\rangle \equiv\left(1-e^{-\nu \tau}\right) l_{n}, l_{0} \equiv v_{0} / \nu, \\
& \sigma_{z}^{2}(\tau) \equiv\left(2 \nu \tau-3+4 e^{-\nu \tau}-e^{-2 \nu \tau}\right) \ell^{2} \\
& r(\tau)=\left(1-e^{-\nu \tau}\right)^{2} D_{\|} / \sigma_{v} \sigma_{z}
\end{aligned}
$$

We show in Appendix A that

$$
D(t)=V_{0} D_{m}^{(0)} d(t)
$$

wliese

$$
\begin{aligned}
D_{m}^{(0)} & \equiv \pi R \delta^{2}, \delta^{2} \equiv \sum_{m} \delta_{m}^{2}, \\
d(t) & \equiv 2 \int_{0}^{t} d \tau \int d V d z P(z, v, \tau) \vee C_{\|}(z) \\
& =\int_{0}^{<z(t)>} d \zeta \int d \bar{z} C_{\|}(\zeta-\bar{z}) P(\bar{z}, t) .
\end{aligned}
$$


Here $P(z, t) \equiv\left[2 \pi \sigma_{z}^{2}(t)\right]^{-1 / 2} \exp \left[-z^{2} / 2 \sigma_{z}^{2}(t)\right]$ is the parallel probability for fluctuations about the mean position. Modeling $C_{\|}(z)$ by the flattop function $\left[H\left(z+L_{0}\right)-H\left(z-L_{0}\right)\right] / 2 L_{0}$, of width $2 \mathrm{~L}_{\mathrm{O}}$ and unit area, one can identify several regimes (Appendix A):

$$
d(t) \sim\left\{\begin{array}{rll}
v_{0} t / L_{0} & \sigma<<z<L_{0} & (v t<1), \\
1 & \sigma, L_{0}<<z> & (v t<1), \\
\ell_{0} / L_{0} & <z>\sigma<L_{0} & (\nu t>1), \\
\ell_{0}\left(\pi D_{\|} t\right)^{-1 / 2} & <z>, L_{0}<\sigma & (\nu t>1) .
\end{array}\right.
$$

Regime $(32 a)$ is a short-time limit in which the lines, and hence the particles, move freely. Regime $(32 \mathrm{~b})$ is the collisionless diffusion limit. Regime $(32 \mathrm{c})$ is an intermediate collisional limit which exists when $\ell_{0} / L_{0}<I$. Regime $(32 d)$ is the collisional, double diffusion limit. RR considered only regimes $(32 b$ and $d)$.

The function $d(\tau)$ and its derivative $d^{\prime}(\tau)$ are plotted in Figs. $1-3$ for various orderings of $L_{0}$ and $l$. We observe in each case the presence of a long, small and negative tail on $d^{\prime}$, of extent such as to make the total area under d' equal to zero. This is related to the radial Lagrangian velocity correlations, as can be seen from the relation $\left\langle v_{r}(\tau) v_{r}(0)\right\rangle=v_{0} D_{m}^{(0)} d^{\prime}(\tau)$. The negative tail is absent both until a significant number of collisions have occurred $(\sigma\rangle\langle z\rangle$, or $v t\rangle 1)$, which produce particles of negative velocities, and until a significant number 
of those particles diffuse radially $\left(\sigma>L_{0}\right)$. In this limit the function $d(t)$, which describes the relative fraction of particles which diffuse radially, decreases as particles diffuse back into the regime of Lagrangian magnetic correlations $|z| \leqslant L_{0}$. The above discussion holds only while particles remain correlated with lines, for $\tau \leqslant \tau_{C}$. For $\tau>\tau_{C}$, $d^{\prime}(\tau)$ goes rapidly to zero and $D \simeq V_{O} D_{m}^{(0)} d\left(\tau_{c}\right)$. The decorrelation process can happen in two ways. Either the lines can "disappear" on a time scale $\tau_{b}$, or particles can leave the lines on a scale $\tau_{\delta}$. Computation of $\tau_{b}$ involves specific properties of the background turbulence and is beyond the scope of this paper. We study the effects of perpendicular transport in the next subsection.

\subsection{Stochastic Broadening and Perpendicular Transport}

We now find approximate solutions to Eqs. (30), which describe the contribution of fluctuations to the integral (25). We study first the homogeneous solution, which gives a collisionless stochastic broadening because of the random directions of the lines. This calculation is equivalent to finding the $\mathrm{K}-$ entropy for the lines, or to solving Eqs. (31).

To define the exponentiation length $L_{K}$, we examine the coefficient of $z$ in the asymptotic expression for the sernnd moment computed from Eq. (3la):

$$
\left\langle\Delta y^{2}(z)\right\rangle \sim\left\langle\Delta y^{2}(0)>\exp \left(2 z / L_{K}\right)\right.
$$

We must, of course, demonstrate that a solution of Eqs. (31) with 
this functional dependence can be found. Let us define

$$
b^{\prime}(y, z) \equiv \partial b / \partial\left(\bar{k}_{\theta} y\right)
$$

Then the second moments of Eqs. (31) obey the exact equations.

$$
\begin{aligned}
& \frac{\mathrm{d}}{\mathrm{dz}}\left\langle\Delta \mathrm{y}^{2}\right\rangle=2 \mathrm{~L}_{\mathrm{s}}^{-1}\langle\Delta \mathrm{y} \Delta \mathrm{r}\rangle, \\
& \frac{\mathrm{d}}{\mathrm{dz}}\langle\Delta \mathrm{y} \Delta \mathrm{r}\rangle=\mathrm{L}_{\mathrm{s}}^{-1}\left\langle\Delta \mathrm{r}^{2}\right\rangle+\overline{\mathrm{k}}_{\theta}\left\langle\Delta \mathrm{y}^{2} \mathrm{~b}^{\prime}\right\rangle, \\
& \frac{\mathrm{d}}{\mathrm{dz}}\left\langle\Delta \mathrm{r}^{2}\right\rangle=2 \overline{\mathrm{k}}_{\theta}\left\langle\Delta \mathrm{r} \Delta \mathrm{y} \mathrm{b}^{\prime}\right\rangle,
\end{aligned}
$$

where $b^{\prime}$ is taken along the exact 1ines. We express the three point functions occuring in Eqs. $(33 b, c)$ in terms of four point ones by integration of Eqs. (31):

$$
\begin{aligned}
\left\langle\Delta y^{2} b^{\prime}\right\rangle & =L_{s}^{-1} \bar{k}_{\theta} \int_{0}^{z} d z^{\prime} \int_{0}^{z^{\prime}} d z^{\prime \prime}\left\langle\Delta y(z) \Delta y\left(z^{\prime \prime}\right) b^{\prime}(z) b^{\prime}\left(z^{\prime \prime}\right)\right\rangle \\
\left\langle\Delta r \Delta y b^{\prime}\right\rangle & =\bar{k}_{\theta} \int_{0}^{z} d z^{\prime}\left\langle\Delta y(z) \Delta y\left(z^{\prime}\right) b^{\prime}(z) b^{\prime}\left(z^{\prime}\right)\right\rangle
\end{aligned}
$$

For $z>L_{0}, \cdots$ the increments of phase $k_{\theta} \Delta y$ should be essentially uncorrelated from the magnetic perturbations. By factoring the four-point averages into products of two and reversing the order of integration in Eq. (34), we obtain 


$$
\begin{gathered}
\left\langle\Delta y^{2} b^{\prime}\right\rangle \simeq \mathrm{L}_{\mathrm{s}}^{-1} \overline{\mathrm{k}}_{\theta} \int_{0}^{\mathrm{z}} \mathrm{dz} \mathrm{z}^{\prime}\left\langle\Delta \mathrm{y}(\mathrm{z}) \Delta \mathrm{y}^{\prime}\left(\mathrm{z}^{\prime}\right)\right\rangle\left\langle\mathrm{b}^{\prime}(\mathrm{z}) \mathrm{b}^{\prime}\left(\mathrm{z}^{\prime}\right)\right\rangle\left(\mathrm{z}^{\prime}-\mathrm{z}^{\prime}\right), \\
\left\langle\Delta \mathrm{r} \Delta \mathrm{y} \mathrm{b}^{\prime}\right\rangle \simeq \overline{\mathrm{k}}_{\theta} \int_{0}^{z} \mathrm{dz} \mathrm{z}^{\prime}\left\langle\Delta \mathrm{y}(\mathrm{z}) \Delta \mathrm{y}\left(\mathrm{z}^{\prime}\right)\right\rangle\left\langle\mathrm{b}^{\prime}(\mathrm{z}) \mathrm{b}^{\prime}\left(\mathrm{z}^{\prime}\right)\right\rangle
\end{gathered}
$$

In this same limit Eq. (35b) can be approximated as

$$
\left\langle\Delta r \Delta y b^{\prime}\right\rangle \simeq \overline{\mathrm{k}}_{\theta} D_{\mathrm{m}}^{\prime \prime}\left\langle\Delta \mathrm{y}^{2}\right\rangle \text {, }
$$

with a similar approximation holding for (35a). Here we have introduced an object $\mathrm{D}_{\mathrm{m}}^{\prime \prime}$,

$$
D_{m}^{\prime \prime} \equiv \int_{0}^{\dot{\omega}} \mathrm{d} \zeta\left\langle b^{\prime}(\zeta) b^{\prime}(\underline{\theta})\right\rangle,
$$

which is of the same order as the usual magnetic diffusion coefficient $D_{m}$. We will find that the characteristic scale in $z$ of Eqs. (33) is $L_{K}$, where $L_{K}$ is defined by Eq. (16). Then if we use the scaling $\Delta y \sim \mathrm{L}_{K} \Delta \mathrm{r} / \mathrm{L}_{S}$, it is easy to see that the term $\left\langle\Delta y^{2} b^{\prime}\right\rangle$ in Eq. (33b) is smaller by a factor of $\mathrm{L}_{\mathrm{O}} / \mathrm{L}_{\mathrm{K}}$ than the term in $\left\langle\Delta \mathrm{r}^{2}\right\rangle$. Neglect of the former term and combination of the resulting equations yields

$$
\frac{\mathrm{d}^{3}}{\mathrm{dz} \mathrm{z}^{3}}\left\langle\Delta \mathrm{y}^{2}\right\rangle=\left(\frac{2}{\mathrm{~L}_{\mathrm{K}}}\right)^{3}\left\langle\Delta \mathrm{y}^{2}\right\rangle
$$

If we assume exponential variations of the form exp(ilz), the characteristic wavenumbers for Eq. (37) are $i \ell=2 L_{K}^{-1}\{1$, $\left.-\frac{1}{2}(1 \pm i \sqrt{3})\right\}$, which indicates one growing solution $\sim \exp \left(2 z / L_{K}\right)$ and two decaying ones $\sim \exp \left(-z / L_{K}\right) \cdot$ Exact solution of Eq. (37) 
for arbitrary initial conditions is straightforward but uninteresting. The asymptotic solution is

$$
\begin{aligned}
\left\langle\Delta \mathrm{Y}^{2}(\mathrm{z})\right\rangle & \sim \frac{1}{3}\left[\left\langle\Delta \mathrm{Y}^{2}(0)\right\rangle+\left(\mathrm{L}_{\mathrm{K}} / \mathrm{L}_{\mathrm{S}}\right)\langle\Delta \mathrm{Y}(0) \Delta \mathrm{r}(0)\rangle\right. \\
& \left.+\frac{1}{2}\left(\mathrm{~L}_{\mathrm{K}} / \mathrm{L}_{\mathrm{S}}\right)^{2}\left\langle\Delta \mathrm{r}^{2}(0)\right\rangle\right] \exp \left(2 \mathrm{z} / \mathrm{L}_{\mathrm{K}}\right) .
\end{aligned}
$$

Equation (37) is formally identical to Dupree's Eq. (72) (Dupree 1972) for the relative motion of two particles in a clump, if we make the identification of variables $z \rightarrow t$, $r \rightarrow v, y \rightarrow x$. This is not surprising, because the particle orbits in weak vlasov turbulence suffer a corresponding stochastic instability. We shall discuss this point further in sec. 5 and 6.

The function $\exp \left[-(1 / 2) k_{\theta}^{2}<\Delta y^{2}(z)>\right]$, which varies with $z$ on the $L_{K}$ scale, provides a fluctuation renormalization of the magnetic diffusion coefficient due to the random directions of the lines. Because it multiplies $\delta\left(\mathrm{z} ; \Delta \mathrm{k} \|^{\prime}\right.$ in Eq. (26) and because $\mathrm{L}_{\mathrm{O}} / \mathrm{L}_{\mathrm{K}}<1$, its numerical effect on the initial decay of correlations is small. It is conceptually important, however, because it provides the envelope of the magnetic Lagrangian correlation function (see Sec. 2.6).

We turn to the inhomogeneous solution of Eqs. (30). The following exact moment equations hold: 


$$
\begin{aligned}
\frac{d}{d \tau}\left\langle\hat{y}^{2}\right\rangle & =2\left(I_{s}^{-1}\langle V \hat{r} \hat{y}\rangle+\left\langle\hat{y} v_{\perp}\right\rangle\right), \\
\frac{d}{d \tau}\left\langle V \hat{r} \hat{y}^{i}\right. & =-v\langle V \hat{r} \hat{y}\rangle+\langle f \hat{r} \hat{y}\rangle+\bar{k}_{\theta}\left\langle V^{2} b^{\prime} \hat{y}^{2}\right\rangle+L_{s}^{-1}\left\langle v^{2} \hat{r}^{2}\right\rangle^{i}+\left\langle V \hat{r} v_{\perp}\right\rangle, \\
& : \\
\frac{d}{d \tau}\left\langle\hat{r}^{2}\right\rangle & =2 \bar{k}_{\theta}\left\langle V b^{\prime} \hat{r}\right\rangle,
\end{aligned}
$$

where we have written the parallel Langevin equation as

$$
\frac{d V}{d \tau}=-\nu v+f(\tau)
$$

Upon integrating Eq. (30a), we find:

$$
\begin{aligned}
\left\langle\hat{Y} v_{\perp}\right\rangle & =\int_{0}^{\tau} d \tau^{\prime}\left\langle\left[L_{S}^{-1} V\left(\tau^{\prime}\right) \hat{r}\left(\tau^{\prime}\right)+v_{\perp}\left(\tau^{\prime}\right)\right] v_{\perp}(\tau)\right\rangle^{\prime} \\
& =D_{\perp},
\end{aligned}
$$

where the first term vanished because $v_{\perp}$ is delta-correlated and $\hat{r}$ is causal. In a similar manner $\langle f \hat{r} \hat{y}\rangle=0$ and

$$
\begin{aligned}
& \left\langle V^{2} b^{\prime} \hat{y}^{2}\right\rangle \simeq \bar{k}_{\theta} L_{s}^{-1}\left\langle V^{2}\right\rangle \int_{0}^{\tau} d \tau^{\prime} \int_{0}^{\tau^{\prime}} a_{\tau^{\prime \prime}}\left\langle V\left(\tau^{\prime}\right) b^{\prime}(\tau) V\left(\tau^{\prime \prime}\right) b^{\prime}\left(\tau^{\prime \prime}\right) \hat{y}(\tau) \hat{y}\left(\tau^{\prime \prime}\right)\right\rangle \\
& \left\langle V b^{\prime} \hat{y} \hat{r}\right\rangle \simeq \bar{k}_{\theta} \int_{0}^{\tau} d_{\tau^{\prime}}\left\langle V(\tau) b^{\prime}(\tau) V\left(\tau^{\prime}\right) b\left(\tau^{\prime}\right) y(\tau) y\left(\tau^{\prime}\right)\right\rangle .
\end{aligned}
$$

Under the usual factorization and Markovian approximations, we arrive at 


$$
\begin{gathered}
\frac{d}{d \tau}\left\langle\hat{y}^{2}\right\rangle=2 L_{s}^{-1}\langle V \hat{r} \cdot \hat{y}\rangle+2 D_{+}, \\
\left.\frac{d}{d \tau}<v \hat{r} \hat{y}\right\rangle=-v\langle V \hat{r} \hat{y}\rangle+L_{s}^{-1}\left\langle V^{2}\right\rangle\left\langle\hat{r}^{2}\right\rangle, \\
\frac{d}{d \tau}\left\langle\hat{r}^{2}\right\rangle=2 \bar{k}_{\theta}^{2} D^{\prime \prime}(\tau)\left\langle\hat{y}^{2}\right\rangle .
\end{gathered}
$$

In the collisionless limit $\nu \tau \ll 1$, the change of variables $z_{0}(\tau) \equiv V_{0} \tau$ reduces Eqs. (39) to the K-entropy problem we have already solved. The nonvanishing initial condition is

$$
d<\hat{y}^{2}(0)>/ d \tau=2 D_{\perp}
$$

and the asymptotic solution is [cf. Eq. (38)]

$$
\left\langle\hat{y}^{2}(\tau)\right\rangle \quad-\frac{1}{3} D_{\perp}\left(L_{K} / L_{0}\right) \exp \left[2 z_{0}(\tau) / L_{K}\right]
$$

In the collisional limit $\nu t>1$, an approximate solution is obtained by balancing the two terms on the right-hand side of $\mathrm{Eq} \cdot(39 \mathrm{~b})$ :

$$
\langle V \hat{r} \hat{y}\rangle \simeq\left(V L_{S}\right)^{-1}\left\langle V^{2}\right\rangle\left\langle\hat{r}^{2}\right\rangle .
$$

The differential equation which results is

$$
\begin{aligned}
& \frac{d^{2}}{d \bar{\tau}^{2}}\left\langle\hat{y}^{2}\right\rangle=\bar{\tau}^{-1 / 2}\left\langle\hat{y}^{2}\right\rangle, \\
& \bar{\tau} \equiv\left[2 z_{C}(\tau) / L_{K}\right]^{2}, \\
& z_{C}(\tau) \equiv\left(2 D_{\|} \tau\right)^{1 / 2} .
\end{aligned}
$$


If we extend the range of validity of $\mathrm{Eq}$. (42a) back to $\bar{\tau}=0$ and apply the boundary conditions (40), the solution of Eq. (42) is

$$
\frac{1}{2} k^{2}\left\langle y^{2}\right\rangle=\left(\frac{2}{3}\right)^{1 / 3} \Gamma\left(\frac{2}{3}\right)\left(\frac{\tau}{\tau_{1}}\right)^{-}-1 / 2 I_{2 / 3}\left(\frac{4}{3} \tau \cdot 3 / 4\right),
$$

where $I_{\nu}$ is the modified Bessel function of the first kind. This procedure is not strictly correct, because Eqs. (42) is valid only for $\nu \tau \gg l$ and the initial conditions should be matched onto the solution of Eq. (39) for $\nu \tau \sim 1$. The matching is not crucial here because we are concerned only with the asymptotic behavior. Since $I_{\nu}(z) \sim(2 \pi z)^{-1 / 2} \exp (z)$, we find that (43) becomes of order one and therefore contributes significantly to the Lagrangian correlation when

$$
\mathrm{L}_{\delta} \equiv \mathrm{z}_{\mathrm{C}}(\tau)=\mathrm{L}_{\mathrm{K}} \ln ^{2 / 3}\left(\ell_{\perp} / \mathrm{L}_{\mathrm{K}}\right)
$$

where $\ell_{\perp} \equiv\left(2 \mathrm{D} \| \tau_{\perp}\right)^{1 / 2}$, the form $(44)$ is valid for $\ell_{\perp} / L_{K}>1$ and where we have dropped a numerical coefficient of $O(1)$. The precise value of the exponent should not be taken too seriously.

In general, we can summarize the contents of Eqs. (41) and (44) by stating that $\frac{1}{2} \mathrm{k}_{\theta}^{2}<\mathrm{y}^{2}>=1$ when

$$
L_{\delta} \equiv z\left(\tau_{\delta}\right) \simeq \alpha: I_{K} \ln ^{\beta}\left(\ell_{\perp} / L_{K}\right)
$$

Here $\alpha$ and $\beta$ are numbers of order unity, $z=z_{0}(\nu \tau<1)$ or $z=z_{C}(\nu \tau>1)$, and we assume $\ell_{1} / L_{K}>1$. Notice that one can 
also write $\ell_{\perp} / L_{\delta}=\left(\tau_{\perp} / \tau_{\delta}\right)^{1 / 2}$.

The correlation time $\tau_{\delta}$ describes the effects of perpendicular transport. For $t<\tau_{\delta}$ the solution of the parallel transport problem is valid. For $t>\tau_{\delta}, d(t)$ levels off at $d\left(\tau_{\delta}\right)$ and defines the diffusion coefficient (9): Mathematically, this behavior follows if we model the factor $\exp \left(-\frac{1}{2} \mathrm{k}_{\theta}^{2}\left\langle\mathrm{Y}^{2}\right\rangle\right)$ by the step function $\delta\left(-\infty, \tau, \tau_{\delta}\right) \fallingdotseq 1-H\left(\tau-\tau_{\delta}\right)$. The time integration in $\mathrm{Eq}$. (A5) is then replaced by

$$
\int_{0}^{t} d \tau \delta\left(-\infty, \tau, \tau_{\delta}\right) \frac{d}{d \tau} e^{F(\tau)}=H\left(\tau_{\delta}-t\right)\left(e^{F(t)}-1\right)+H\left(t-\tau_{\delta}\right)\left(e^{F\left(\tau_{\delta}\right)}-1\right) .
$$

Let us note that

$$
v \tau_{\delta} \simeq \begin{cases}\mathrm{L}_{\mathrm{K}} / \ell & , \quad \nu \tau_{\delta}<1 . \\ \left(\mathrm{L}_{\mathrm{K}} / \ell\right)^{2}, & \nu \tau_{\delta}>1 .\end{cases}
$$

We can thus define the collisionless (collisional) regimes by $\mathrm{L}_{\mathrm{K}} / \ell<I\left(\mathrm{~L}_{\mathrm{K}} / \ell>1\right)$, in agreement with $\mathrm{RR}$. There are apparentIY no interesting intermediate regimes of $\mathrm{L}_{\mathrm{K}} / l$.

There are interesting differences between the present calculation and the heuristic arguments of RR. First, we have succeeded in finding a simple integral expression (A5) which describes the dynamics of the parallel transport problem. We can thus describe transitions between collisionality regimes and treat a variety of spectra. The procedure of RR predicted asymptotic results only and further did not describe the regime $\ell / L_{0}<1$. Second, by writing $D \propto d\left(\tau_{c}\right)$ and allowing the 
possibility. $\tau_{c}=\tau_{b}$, we embrace the situation of a time-dependent background not discussed by RR. Finally, our calculation is an attempt at a derivation beginning from first principles. We address this important aspect further in the next section.

\section{Renormalized Kinetic Theory}

We now formulate the particle transport problem in terms of kinetic equations for the averaged particle distribution function. This approach is useful for two reasons. First, it provides a convenient systematology by which one can attempt to justify the heuristic procedures of Sec. 4. Second, it is readily generalized to the self-consistent problem. The formal techniques which we employ lead to quite complicated nonlinear equations which, however, reduce readily to workable practical approximations. The procedure, based on a renormalized stochastic Liouville equation ${ }^{\dagger}$ for the test particles, brings us into close contact with modern advances and insights of turbulence theory.

The most important observation in this section is that it is both necessary and natural to introduce the joint probability $k$ for the combined system of particles and field. Such an object is, of course, implicit in the conventional renormalized QLT, which factors. $K$ into the product of the particle probability times the field correlation function. However, this approximation is in general incorrect because it misses the exponential

\footnotetext{
${ }^{A}$ good review with many references is Van Kampen (1976).
} 
divergence of adjacent lines. It thus erroneously replaces ${ }^{\tau} \delta$ by $\tau_{\mathcal{L}} \gg \tau_{\delta}$, therefore errs if $\tau_{b}>\tau_{\delta}$. The approximation which we discuss is a systematically renormalized generalization of QLT which retains phase information sufficient to describe the exponential divergence. It is philosophically related to Dupree's theory of clumps though our approach is different and our techniques more systematic.

We consider the stochastic Liouville function

$$
\tilde{\mathrm{P}}(r, \theta, \phi, V, t) \equiv \delta[r-\tilde{r}(t)] \delta[\theta-\tilde{\theta}(t)] \delta[\phi-\tilde{\phi}(t)] \delta[V-\tilde{\mathrm{V}}(t)],
$$

whose average $\langle\tilde{\mathrm{p}}\rangle$ defines the test particle probability density in the guiding center phase space. This function obeys

$$
\left.\frac{\partial \tilde{P}}{\partial t}+V \hat{n} \cdot \underset{\sim}{\nabla} \tilde{P}+\underset{\sim}{V_{\perp}}(t) \cdot{\underset{\sim}{\perp}}_{\perp} \tilde{P}+\frac{\partial}{\partial V}\left\{f f \|^{(t)}-v V\right] P\right\}=0,
$$

which describes Brownian particle motion along the stochastic field lines $\underset{\sim}{B}=\hat{n} B$ with simultaneous diffusion across $B$. We will treat $\underline{\sim}_{\perp}, \mathrm{f}_{\|}$, and the radial perturbation $b$ as given random processes. Now Krommes and Smith (1976) as well as many others (Van Kampen 1976; Frisch 1968) have pointed out that equations of this kind are formally identical to the well-known stochastic oscillator equation

$$
\partial_{t} \psi=-i \omega(t) \psi(t)
$$


introduced by Kraichnan (196I) for time-independent $\omega$ (Gaussianly distributed and independent of $\psi$ ) "to illustrate the problems, successes, and failures of renormalized turbulence theory. The technical problem with equations of this kind is that the evolution equation for $\langle\psi\rangle$ is not trivially closed, but rather involves the two-point function $\langle\omega \psi\rangle$, This kind of coupling in a formally linear equation (in the dependent variable $\psi$ ) is called stochastic nonlinearity to distinguish it from the dynamically nonlinear $\psi^{2}$ coupling in, say, the vlasov equation. Techniques at all levels of sophistication have been advanced for constructing well-behaved equations for $\langle\psi\rangle$. Often the equation is formally iterated to all orders in the stochastic coupling, averaged assuming Gaussian initial conditions and random coefficients, and then partially resummed (Kraichnan 1961; Frisch 1968). Because of the complicated branching properties of the equation under iteration, the terms in the perturbation expansion are often represented diagrammatically. Though tedious and physically opaque, the diagrammatic approach is tractable at lowest nontrivial order, which corresponds to Kraichnan's Direct Interaction Approximation (DIA) for turbulence (Kraichnan 1958). However, its direct application to higher order so obscures the physics as to be questionable. We shall employ instead the renormalization procedure of Martin, Siggia, and Rose (MSR) (1973); who used functional analysis to represent infinite subseries of primitive diagrams in closed form. We stress that this approach is equivalent to, but much more succinct and therefore more useful than, the diagrammatic approach. For completeness and pedagogy, we 
review aspects of the diagrammatic procedure in Appendix $B$.

To properly treat $b$ as a random variable, one must understand that $b$ depends both on coordinates $q \equiv\{r, \theta, \phi, v\}$ and on its Fourier amplitudes $\left\{\delta_{m n}\right\}: b=b(q ;\{\delta\})$. It is the Fourier amplitudes $\{\delta\}$, and only those, which cause $b$ to be random. As in Sec. 4, we usually think of $\{\delta\}$ as being Gaussian. Even if the $\{\delta\}$ were fixed, however, they can still induce stochasticity by island overlap. The important point is that $b$ is not $a$ functional of the random forces $v_{\perp}, f_{\|}$, the effects of which are contained in the random orbits $\tilde{q}(t)$, not in the observation variables $q$. We can thus average in two steps, first over the collisional randomness, later over the effects of stochasticity. We assume that $\underset{\sim}{\mathrm{V}_{\perp}}$ and $\mathrm{f}_{\|}$are white noise processes, characterized by their autocorrelations

$$
\begin{aligned}
& \left\langle\underset{\sim}{v_{\perp}}(t+\tau) \underset{\sim}{v_{\perp}}(t)\right\rangle=2 D_{\perp} \delta(\tau) \underset{\approx}{l_{\perp}}, \\
& \left\langle f_{\|}(t+\tau){\underset{f}{\|}}_{\mid}(t)\right\rangle=2 D_{v} \delta(\tau) .
\end{aligned}
$$

Then one can show that Eq. (46) averaged over only the collisional randomness becomes

$$
\frac{\partial P}{\partial t}+V \hat{n} \cdot \nabla P-D_{\perp} \nabla_{1}^{2} P-\frac{\partial}{\partial V}\left[\left(u V+D_{v} \frac{\partial}{\partial V}\right) P\right]=0
$$

This result is an example of a theorem on Markov processes, discussed for example by Van Kampen (1976). If we change variables 
from $(\dot{\theta}, \phi)$ to $(y, z)$ and make the usual expansion of small deviation around a rational surface $r_{0}$, we reduce Eq. (47) to oúr final stochastic equation:

$$
\frac{\partial P}{\partial t}+V\left(\frac{\partial}{\partial z}+\frac{\left(r-r_{O}\right)}{L_{S}} \frac{\partial}{\partial y}\right) P-D_{\perp} \frac{\partial^{2}}{\partial y^{2}} P-C P-V b \frac{\partial}{\partial r} P=\underset{(48)}{0}
$$

Here $\mathrm{C}$ is the velocity space collision operator, the last term of Eq. (47). We have retained only the azimuthal part of the perpendicular Laplacian, because the effect of the stochastic term $\propto b$ will be the stochastic diffusion $\partial_{r} D \partial_{r}$ and we assume always that $D \gg D_{\perp}$. (Note that we are not, for the moment at least, severely restricted in our choice of the collision operator. We could, for example, employ instead for $C$ the dichotomic Markov operator (Van Kampen 1976) which treats the paralle1 velocity as a two-state (forward or backward) process. $P$ would then be a two-component vector. 1) Finally, to stress the generic nature of the stochastic equation (43), we write it symbolically as

$$
\left(\partial_{t}+i L\right) P=-i \omega P
$$

where the random "frequency" operator. $\omega$ is defined as

$$
i \omega \equiv \mathrm{V} b \frac{\partial}{\partial r}
$$

and the coherent operator il is obvious.

$\dagger_{G}$. Laval has taken this approach in his study of the zero shear problem (private communication, 1977). 
There are several ways of operationally defining the diffusion cojefficient. The way which generalizes most readily to the self-consistent problem is the following. We assume that at $t=t_{0}$ (far in the past) the test particle is in thermal equilibrium-- a velocity-space Maxwellian uniform over all space. The time $t_{0}$ is called the preparation time of the system. Beginning at $t_{0}$, we apply complicated (and unspecified) forces to the system such that at $t=t^{\prime}$ the test particle probability is infinitesimally functionally perturbed from the equilibrium state. The precise form of the perturbation, in particular its radial dependence, is unimportant as long as it is small. At time $t^{\prime}$, we remove the driving forces and allow the system to regress freely to thermal equilibrium. If the equation for the time rate of change of the perturbation in the averaged spatial probability density $\delta \mathrm{n} \equiv \rho \mathrm{dV} \delta<\mathrm{P}>$ includes a term of the form $\partial_{r} D \partial_{r} \delta n$, then $D$ may be defined as the radial diffusion coefficient. ${ }^{+}$

We shall denote averages over the stochasticity by $\langle\bullet$. More specifically, the bracket operation signifies a linear functional operation $A$ on its arguments such that $A^{2}=A$. Many such linear functionals exist. We shall employ an operator which treats the Fourier amplitudes $\delta$ of the angle variables as though they were Gaussian. More specifically, consider an arbitrary (periodic) function $F\left(\Theta_{1}, \Theta_{2}, \ldots, \Theta \Theta_{n}\right)$ of $n$ generic angles (i) and actions $J_{i}$ (in our problem, $\Theta \equiv\{\theta, \phi\}$ ). The average of

${ }^{A}$ A similar approach is taken, for example, by Kadanoff and Martin (1963). 
$F$ is defined in terms of its Fourier decomposition, with respect to the angle variables only, as

$$
\left\langle F\left(\Theta_{1} \cdots \Theta_{n}\right)\right\rangle \equiv m_{1} \sum m_{n}<\delta_{m_{1}} \delta_{m_{2}} \ldots \delta_{m_{n}}>\exp \left(i \sum_{i=1}^{n} m_{i} \Theta_{i}\right),
$$

where the $\mathrm{n}$ point average is interpreted as the sum over the $\mathrm{n}$ !! distinct permutations of products of averages taken two at a time ( $n$ even) or as zero ( $n$ odd), and where

$$
\left\langle\delta_{m_{1}}\left(J_{1}\right) \delta_{m_{2}}\left(J_{2}\right)\right\rangle \equiv\left\langle\delta_{m_{1}}\left(J_{1}\right) \delta_{-m_{1}}\left(J_{2}\right)>\delta_{m_{1},-m_{2}} .\right.
$$

As an example,

$$
\left\langle F\left(\Theta_{1}, \Theta_{2}\right)\right\rangle=\sum_{m}\left\langle\delta_{m}\left(J_{1}\right) \delta_{-m}\left(J_{2}\right)\right\rangle \exp \left[\operatorname{im}\left(\Theta_{1}-\Theta_{2}\right)\right] .
$$

We stress that the prescription is also appropriate for the case where the Fourier amplitudes are in fact not Gaussian, but rather constant (provided islands overlap), because of the mixing properties in angle expected for the stochastic field in the limit of large stochasticity. Indeed, the result (49) can be interpreted as the angle-averaging procedure (Krommes \& Smith 1976)

$$
\left\langle F\left(\Theta_{1}, \Theta_{2}\right)\right\rangle=\int_{0}^{2 \pi} \frac{\mathrm{d} \bar{\theta}}{2 \pi} \mathrm{F}\left(\Theta_{1}-\Theta_{2}+\bar{\theta}, \bar{\theta}\right) .
$$


We now show that we can rigorously shift our attention from general perturbations around equilibrium to an averaged Green's function, a useful object in analytic theories of turbulence (Orszag 1977). [The following discussion will be familiar to workers in the field of turbulence and transport. We include it in an attempt to make the report reasonably self-contained. Experts can jump immediately to the paragraph preceding Eq. (61).] Consider the function

$$
P_{+}\left(\underset{\sim}{1}, t ; t^{\prime}\right) \equiv H\left(t-t^{\prime}\right) P\left(\underset{\sim}{1}, t ; t^{\prime}\right),
$$

where $H$ is the Heaviside function. This causal function satisfies

$$
\left(\partial_{t}+i L+i \omega\right) P_{+}\left(\underset{\sim}{1}, t ; t^{\prime}\right)=\delta\left(t-t^{\prime}\right) P\left(\underset{\sim}{1}, t^{\prime} ; t^{\prime}\right),
$$

where

$$
\left\langle P\left(\underset{\sim}{1}, t^{\prime} ; t^{\prime}\right)\right\rangle=P_{e q}(\underset{\sim}{1})+\varepsilon \delta P(\underset{\sim}{1}),
$$

$\varepsilon$ is infinitesimal, and $\delta \mathrm{P}(\underset{\sim}{1})$ is the shape of the perturbation away from equilibrium. By averaging Eq. (50), we obtain

$$
\left.\left.\partial_{t}<\Delta \mathrm{P}_{+}>+i \mathrm{~L}<\Delta \mathrm{P}_{+}\right\rangle+i \Delta\left\langle\omega \mathrm{P}_{+}\right\rangle=\varepsilon \delta\left(t-t^{\prime}\right) \delta \mathrm{P}_{(1}^{1}\right)
$$

where the equilibrium part has cancelled and $\langle\mathrm{P}\rangle=\mathrm{P}_{\text {eq }}+\left\langle\Delta \mathrm{P}_{+}\right\rangle$. Notice that the equation is not yet closed for $\left\langle\Delta \mathrm{P}_{+}\right\rangle$because of the stochast.ic nature of $\omega$. Furthermore, in general $\omega$ is a 
functional -- usually linear-- of $\Delta \mathrm{P}_{i_{+}}$so that $\left\langle\Delta \mathrm{P}_{+}\right\rangle$contains, in general, terms of higher order in $\varepsilon$ than one. The part $\left\langle\delta P_{+}\right\rangle$proportional to $\varepsilon$ obeys

$\left.\partial_{t}<\delta P_{+}>+i L<\delta P_{+}>+i<(\delta \omega) P_{+e q}+\omega\left\{P_{+e q}\right\} \delta P_{+}>=\delta\left(t-t^{\prime}\right) \delta P_{(\sim}^{(1)}\right)$

The solution of Eq. (51) is

$$
\left\langle\delta P_{p}\left(\underset{\sim}{1}, t ; t^{\prime}\right)\right\rangle=\int d \underset{\sim}{I^{\prime}}\left\langle\hat{R}\left(\underset{\sim}{1}, t ; \underset{\sim}{I^{\prime}}, t^{\prime}\right) \delta P\left(\underline{\sim}^{\prime}\right)\right\rangle
$$

where the stochastic infinitesimal response function $\ddot{R}$ is defined as the functional derivative

$$
\hat{R}\left(1,1^{\prime}\right) \equiv \delta P(1) / \delta n_{-}\left(1^{\prime}\right),
$$

and where $P(1)$ is the causal solution to

$$
\left(\partial_{t}+i L+i \omega\right) P=n:(1)
$$

Here $\eta_{\text {.. }}$ is a non-random source. In other words, $\hat{k}$ is the stochastic Green's function for Eq. (50). It describes the future motion of the test particle, given precise knowledge $\delta\left(\underset{\sim}{1}-\underset{\sim}{1}{ }^{\prime}\right)$ of its state at $t=t^{\prime}$. In the present case where $\delta \omega$ vanishes, $\hat{R}$ is independent of the perturbation $\delta \mathrm{P}$, so the average in Eq. (52) factors to yield 


$$
\left\langle\delta P_{+}\left(\underset{\sim}{I}, t ; t^{\prime}\right)\right\rangle=\int d \underset{\sim}{I^{\prime}} R\left(\underset{\sim}{I}, t ; \underset{\sim}{I^{\prime}}, t^{\prime}\right) \delta P\left(I_{\sim}^{\prime}\right)
$$

where $R \equiv\langle\hat{R}\rangle$ is the averaged infinitesimal response function. Clearly, the evolution of $R$ is not influenced by the form of the radial perturbation, though it still contains information about the diffusion. In a translationally invariant system, $R$ will be a function of only the difference coordinate $\rho \equiv r-r^{\prime}$. (This is nearly always true locally, over distances large compared to the scales for stochastic transport but small compared to the density scale length.) If the equation of motion for the Fourier transform of the radial density $n_{\ell} \equiv \int d V d y d z R$ can be written in the form

$$
\partial_{\tau} \mathrm{n}_{\ell}+\ldots+\ell^{2} \mathrm{D} \mathrm{n}_{\ell}=0 \quad(\tau>0)
$$

in the limit $\ell \rightarrow 0$, then $D$ is the diffusion coefficient. We turn to the solution of the closure problem-- namely, the task of expressing the average $\langle\omega \hat{R}\rangle$ in terms of $R$ and possibly other measurable (at least in principle) two point averages (observables). It is useful to motivate the general discussion by first examining the first few terms of perturbation theory. We do this in Appendix B. There, we introduce a standard diagrammatic technique and show how to resum the formal perturbation theory solution of Eqs. $(53,54)$ in powers of $\omega$ to give either renormalized QLT or one more complicated renormalization. 
Now it is possible, though cumbersome at best, to extend these techniques to more and more complicated summations. In a fundamental though somewhat opaque paper, Kraichnan (1961) has discussed a number of renormalizations, including one very successful one, his so-called "second stochastic model." . The relative diffusion equations which we shall develop shortly are closely related to the second stochastic model. Because of the complexity of the graphs, the physical interpretation of this approximation was obscure. However, the entire procedure becomes clear, to all orders, by the esthetically pleasing and operationally compact functional renormalization scheme of MSR. Krommes (1977) has discussed the relation of the MSR formalism to several problems of interest in plasma physics.

MSR considered evolution equations, for the stochastic variable $\psi$, with quadratic (dynamic) nonlinearity:

$$
\partial_{t} \psi(1)=U(1,2) \psi(2)+\frac{1}{2} \bar{U}(1,2,3) \psi(2) \psi(3) .
$$

Here the bare interaction vertices $U, \bar{U}$ are specified and nonrandom, and the usual integration convention over repeated indices is assumed. Deker and Haake (1975) extended the formalism to the case of present interest by adding the stochastically nonlinear term $U^{\prime}(1,2,3) i \omega(2) \psi(3)$. In our general discussion, we shall lump both of these cases together. Consider then the symmetric matrix $G$ of two-point functions 


$$
G\left(1, I^{\prime}\right) \equiv\left(\begin{array}{ccc}
C\left(1,1^{\prime}\right) & R\left(1,1^{\prime}\right) & M\left(1,1^{\prime}\right) \\
R^{\operatorname{Tr}}\left(1,1^{\prime}\right) & 0 & 0 \\
M^{\operatorname{Tr}}\left(1,1^{\prime}\right) & 0 & F\left(1,1^{\prime}\right)
\end{array}\right) \text {, }
$$

where

$$
\begin{aligned}
& C\left(1,1^{\prime}\right) \equiv\left\langle\delta \psi(1) \delta \psi\left(1^{\prime}\right)\right\rangle, \\
& M\left(1,1^{\prime}\right) \equiv\left\langle\delta \psi(1) \delta\left[i \omega\left(1^{\prime}\right)\right]\right\rangle, \\
& F\left(1,1^{\prime}\right) \equiv\left\langle\delta[i \omega(1)] \delta\left[i \omega\left(1^{\prime}\right)\right]\right\rangle,
\end{aligned}
$$

and "Tr" denotes transpose. The goal is to derive closed (functional) equations for these objects and the averaged field $\langle\psi\rangle$. To do this, MSR began by introducing the operator

$$
\hat{\psi}(1) \equiv-\frac{\partial}{\partial \psi(1)},
$$

taken in the Heisenberg representation (Rose 1974; Phythian 1975), and considering the generating functional (DeDominicis 1964)

$$
s\{\eta\} \equiv\left\langle\exp _{\mathrm{T}}[\Phi(1) \eta(1)]\right\rangle,
$$

where

$$
\begin{aligned}
& \Phi \equiv(\psi, \hat{\psi}, i \omega), \\
& n \equiv\left(n_{+}, n_{-}, n_{0}\right),
\end{aligned}
$$


and "T" denotes time ordering. Two point cumulants of the elements of $\Phi$ follow by functional differentiation of the logarit:hm of $S$. For example,

$$
\begin{aligned}
& \langle\psi(1)\rangle=\left.\frac{\delta}{\delta \eta_{+}(1)} \ln s\right|_{\eta=0} . \\
& c\left(1,1^{\prime}\right)=\left.\frac{\delta}{\delta n_{+}(1)} \frac{\delta}{\delta \eta_{+}\left(1^{\prime}\right)} \ln s\right|_{\eta=0} .
\end{aligned}
$$

One can show that the averaged infinitesimal response function $\mathrm{R}$ is given by

$$
\begin{aligned}
R\left(I, I^{\prime}\right) & =\left\langle\psi(1) \hat{\psi}\left(I^{\prime}\right)\right\rangle_{\mathrm{T}} \\
& =\left.\frac{\delta}{\delta n_{+}(1)} \frac{\delta}{\delta n_{-}\left(I^{\prime}\right)} \ln S\right|_{11-0},
\end{aligned}
$$

so that: $G$ is properly called the correlation matrix:

$$
G\left(1, I^{\prime}\right)=\frac{\delta^{2}}{\delta n(1) \delta n\left(I^{\prime}\right)} \text { ln }\left.s\right|_{n=0} \text {. }
$$

Relations between the two-point functions follow by examining the functional variations with respect to $n$ of the two point cumulants at finite $\eta$. MSR have discussed the physically interesting case of Gaussian initial conditions and random forces. For this case one can write the matrix Dyson equation

$$
G_{0}^{-1} G-\gamma<{ }_{0}>G-\Sigma G=1
$$


represented diagrammatically in Fig. 4a. The mass or "collision" operator $\Sigma$ is defined in terms of the two-body scattering matrix $\mathrm{K}(1,2 ; 3,4):^{\dagger}$

$$
\sum(1, \overline{1})=\frac{1}{2} \gamma(1,2,3) \mathrm{K}(2,3 ; \overline{2}, \overline{3}) \gamma(\overline{2}, \overline{3}, \overline{1})
$$

(Fig. 4b). K obeys the Bethe-Salpeter equation (Frisch 1968; Krommes \& Smith 1976; Krommes 1977)

$$
\begin{aligned}
& K(1,2 ; 3,4)=\frac{1}{2}[G(1,3) G(2,4)+G(1,4) G(2,3)] \\
& \quad+G(1, \overline{1}) G(2, \overline{2}) I(\overline{1}, \overline{2} ; \overline{3}, \overline{4}) K(\overline{3}, \overline{4} ; 3,4)
\end{aligned}
$$

(Fig. 5a). The interaction kernel I, defined formally as

$$
I(1,2 ; 3,4) \equiv \frac{\delta \sum(1,2)}{\delta G(3,4)},
$$

is the formal expression of the intrinsic two-body correlations. The appearance of the two-body function $\mathrm{K}$ in Eq. (56) is closely related to the fact that diffusion coefficients are most conveniently couched as integrals of Lagrangian correlation functions (Taylor 1921). Kraichnan (1964a) was one of the first to attempt a renormalized description which related a Lagrangian function to Eulerian ones.

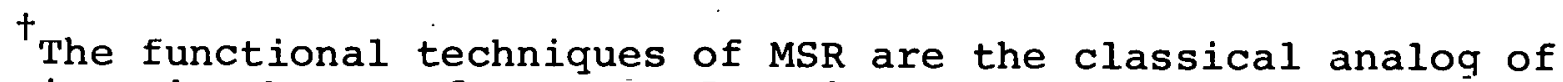
Schwinger's theory of Green's functions for quantum fields. These are reviewed, for example, in the readable book by Nishijima (1969). 
To date, reduction of the functional derivative (58) to practical form has been accomplished only through perturbation theory of some sort. Direct expansion in powers of the bare interaction $\gamma$ defeats the purpose of renormalization and leads to unphysical divergences (Kraichnan 1961). A better procedure (MSR 1973) is to expand $I$ in powers of the renormalized vertex $\Gamma \equiv \mathrm{G}^{-2} \mathrm{~K} \gamma$. (Figs. 4c,d) (more precisely, in powers of $\bar{\Gamma} \equiv \mathrm{G}^{3 / 2} \Gamma$ ), yielding to lowest order

$$
I(1,2 ; 3,4) \simeq \Gamma(1,5,3) \mathrm{G}(5, \overline{5}) \Gamma(2, \overline{5}, 4)
$$

(Fig. 5b). The argument here is that $\bar{\Gamma} \sim\left\langle\delta \psi^{3}\right\rangle /\left\langle\delta \psi^{2}\right\rangle^{3 / 2}$ is a generalized skewness parameter (really an operator). For mixing systems, it is hoped that the actual (measurable) skewness is small (less than one), even though the "bare" skewness $\mathrm{G}^{3 / 2} \gamma$ need not be small. This must, of course, be verified either experimentally or analytically. Both tasks are formidabie, in general. It is especially important to note that $\bar{\Gamma}$ need not be uniformly small over all ranges of its arguments.

For small $\bar{\Gamma}$ it appears justifjed to neglect the interaction term GGIK in Eq. (57). The resulting approximation,

$$
\left[(1, \bar{I}) \simeq \frac{1}{2} \gamma(1,2,3) G(2, \overline{2}) G(3, \overline{3}) \gamma(\overline{3}, \overline{2}, \overline{1})\right.
$$

(Fig. 4e), is called the Direct Interaction Approximation (DIA) after Kraichnan's original derivation (1958) for the NavierStokes equation. For linear stochastic differential equations, 
this reduces to renormalized QLT. When, in addition, the generalized Reynolds number is uniformly small, the theory reduces further to the well-known Bourret approximation (Bourret 1962; Van Kampen 1976; Frisch 1968).

In the present problem $\bar{\Gamma}$ is not uniformly small, first because $\gamma$ is a differential operator which acts on radial structures of all scales, second because of the stochastic instability with which are associated very specific, non-Gaussian correlations responsible for the exponential divergence of lines. The first vertex renormalization $(59,57)$ is a kind of renormalized "Boltzmann" equation, to use the terminology of many-body physics, and the classical Boltzmann operator [Balescu 1975 (pp. 620-25)] would follow from this order (but not from DIA). If we further restrict our attention to the regime of weak nonlinearity, then it is presumably appropriate to pass to the diffusive approximation $I \simeq \gamma G \gamma$ (Fig. 5c). This conclusion is not trivial. Indeed, when this procedure is applied to the stochastic oscillator at infinite Reynolds number (Kraichnan 1961) (admittedly a very severe test), the solution diverges. Presumably, this trouble would show up in the present problem for shear so weak that radial correlations self-consistently dominated the transport. We do not consider this limit, for which the renormalized QLT may again provide an adequate description. In any case, theories based on Fig. 5c are best believed, at the present time, by their favorable comparison with the heuristic analyses of Sec. 4. That we do find agreement provides an interesting motivation for more systematic 
studies of the renormalized theory. We plan to return to this question in future work.

To be more specific, let us represent the magnetic correlations by a jagged line, and the particle response function by a line wiggly at the measurement end and straight at the perturbation end (Fig. 6). The joint probability $\mathrm{K}$ for one particle and the field then satisfies the renormalized approximation represented in Fig. 7. It is convenient to rewrite this as a differential equation by multiplying by $\mathrm{K}^{-1}$ :

$$
\begin{aligned}
& \frac{\partial}{\partial \tau_{2}} K\left(\underset{\sim}{1}, \tau_{1}, \underset{\sim}{2}, \tau_{2} ; \underset{\sim}{3}, \underset{\sim}{4}\right)+[i L(2)-\Sigma(2)] K=\frac{1}{2} F\left(\underset{\sim}{1}, \underset{\sim}{3}, \tau_{1}\right) \delta(\underset{\sim}{2}-\underset{\sim}{4}) \delta\left(\tau_{2}\right)
\end{aligned}
$$

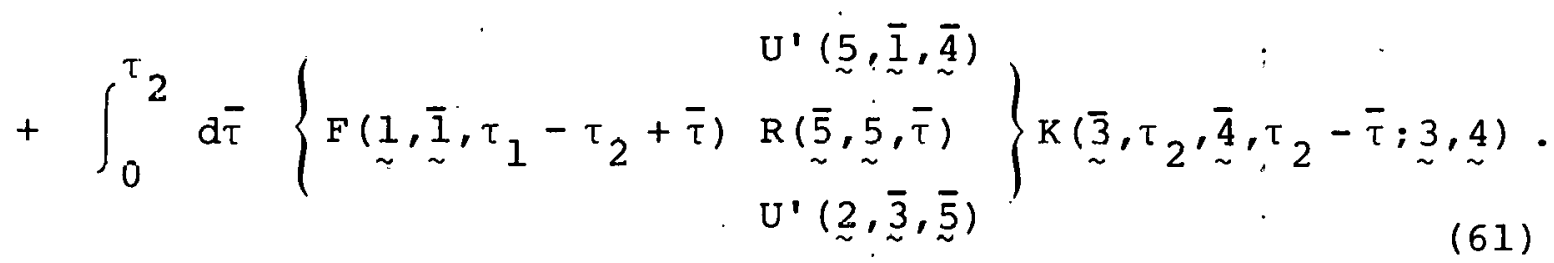

The temporal behavior of this equation is complicaled. However, if temporal fluctuations of the field are ignored, the solution of Eq. (6].) involves no dependence on the first time argument. Explicitly, the $\Sigma K$ term plus the last (interaction) term of Eq. (6].) then becomes

$$
\int_{0}^{\tau} d \tau \int d \bar{\sim} \frac{\lambda}{\partial r_{2}} v_{2} R(\underset{\sim}{2}, \bar{\sim}, \bar{\tau}) v_{2}\left[F(\underset{\sim}{2}, \bar{\sim}) \underset{\sim}{\partial r_{2}} K(\underset{\sim}{1}, \overline{2}, \tau-\bar{\tau}, \underset{\sim}{3}, \underset{\sim}{1})+(1 \leftrightarrow \nu)\right] .
$$

The symmetry of the bracketed terms should be noted. The last term of Eq. (62) should be compared to the last term of (B3b). It is clear that the $\tilde{K}$ of (B3) consists of the first two terms 
in the iteration of $\mathrm{Eq} .(61)$.

For computation of the diffusion coefficient, we will need only the restricted function $K(1,2 ; \overline{1}, \overline{1})$, which can be considered as a function of the difference spatial coordinates $\underset{\sim}{\rho} \equiv \underset{\sim}{x_{1}}-x_{\sim}$, $\stackrel{\sim}{2}_{2} \equiv \mathrm{x}_{2}-\mathrm{x}_{\sim} \bar{\sim}^{\prime}$. If we pass to the Markovian limit in space, we arrive at the interesting kinetic equation

$$
\begin{aligned}
& \frac{\partial}{\partial \tau} \mathrm{K}\left(\stackrel{\sim}{\rho}_{1}, \rho_{2}, \mathrm{~V}, \tau ; \mathrm{V}^{\prime}\right)+\mathrm{V}\left(\frac{\partial}{\partial \mathrm{z}_{2}}+\frac{\rho_{2}}{\mathrm{~L}_{s}} \frac{\partial}{\partial \mathrm{y}_{2}}\right) \mathrm{K}\left(\underset{\sim}{1}, \underset{\sim}{2}, \mathrm{~V}, \tau ; \mathrm{V}^{\prime}\right) \\
& -\left(D_{\perp} \frac{\partial^{2}}{\partial y_{2}^{2}}+C\right) K\left(\underset{\sim}{1}, \underset{\sim}{2}, V, \tau ; V^{\prime}\right) \\
& \text { 1. } \frac{\partial}{\partial \rho_{2}} \int d \bar{V} \tilde{D}(V, \tau ; \bar{V}) \frac{\partial}{\partial \rho_{2}} K\left(\underset{\sim}{1}, \underset{\sim}{2}, \bar{V}, \tau ; V^{\prime}\right)
\end{aligned}
$$

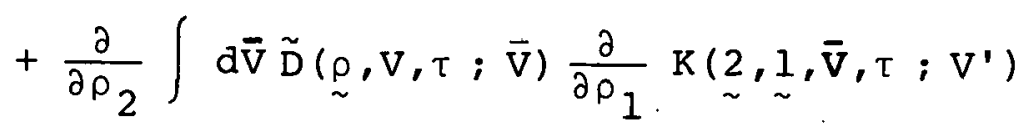

$$
\begin{aligned}
& =\frac{l}{2} F(\underset{\sim}{\rho}) \delta\left(\sim_{2}\right) \delta\left(V-V^{\prime}\right) \delta(\tau),
\end{aligned}
$$

where $\underset{\sim}{\rho} \equiv \rho_{2}-\rho_{1}$,

$$
\begin{aligned}
& \tilde{\mathrm{D}}(\underset{\sim}{\rho}, \mathrm{V}, \tau ; \overline{\mathrm{V}}) \equiv \int_{0}^{\tau} \mathrm{d} \vec{\tau} \int \mathrm{d} \bar{\rho} \sum_{\mathrm{m}} \mathrm{R} \int \mathrm{dk}_{\|} \mathrm{F}_{-\mu} \exp \left[-i\left(\mathrm{k}_{\|} z+\mathrm{k}_{\theta} y\right)\right] \\
& x \cdot V R_{\mu}(\bar{\rho}, V, \bar{\tau} ; \bar{v}) \bar{v} \\
& \tilde{\mathrm{D}}(\mathrm{V}, \tau ; \overline{\mathrm{V}}) \equiv \tilde{\mathrm{D}}(0, \mathrm{~V}, \tau ; \overline{\mathrm{V}}) \text {. }
\end{aligned}
$$

We observe that in the present approximation the diffusion operators $\tilde{D}$ are defined by the factorization $K \simeq R F$. Because the 
solution for the single particle propagator $R$, which obeys

$$
\frac{\partial}{\partial \tau} R\left(\underset{\sim}{\rho}, V, \tau ; V^{\prime}\right)+i L R-D \frac{\partial^{2}}{\partial \rho^{2}} R \underset{\vdots}{=} \delta(\underset{\sim}{\rho}) \delta\left(V-V^{\prime}\right) \delta(\tau)
$$

contains only the term $\exp \left(-\tau / \tau_{\perp}\right)$ involving $D_{\perp}$, the time integrations which define $\tilde{D}$ are not cut off by the exponentiation effect. This lack of self-consistency is not serious, since we need $K$ only up to the cutoff time. If necessary, we could remedy the problem by returning to Eq. (59).

It is convenient to introduce as new spatial variables the relative coordinate $\stackrel{\rho}{\rho}$ and the field coordinate $\stackrel{\rho}{I}_{i} \equiv \rho_{\mathcal{I}}$ :

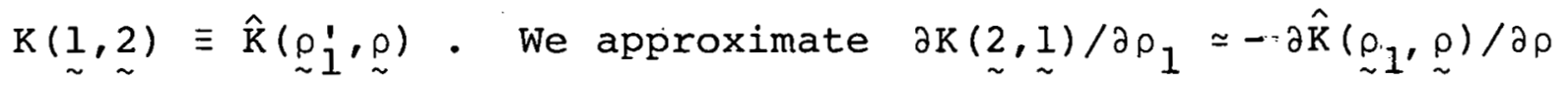
and obtain from Eq. (63)

$$
\begin{aligned}
& \frac{\partial}{\partial \tau} \hat{K}+v\left(\frac{\partial}{\partial z}+\frac{(\rho+\rho)}{L_{s}} \frac{\partial}{\partial y}\right) \hat{K}-\left(D_{\perp} \frac{\partial^{2}}{\partial y^{2}}+C\right) \hat{K}
\end{aligned}
$$

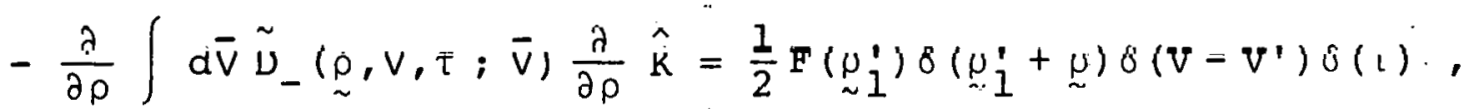

where

$$
\tilde{D}_{-}(\rho) \equiv \tilde{D}-\tilde{D}(\underset{\sim}{\rho})
$$

As can be seen from the form of Eq. (64), the scale for $y$ variation of $\ddot{D}(\rho, v, \tau ; \bar{v})$ is the perpendicular correlation length of the field, of order the perpendicular wavelength $\mathrm{r} / \mathrm{m}$. We assume and verify a posteriori that the cutoff due to the exponential divergence (which we shall rederive momentarily) occurs before 
particles have diffused a perpendicular correlation length. Expansion of $(64 a)$ in small $y$ is then appropriate. The firstorder term vanishes by symmetry, so

$$
\tilde{D}_{-}(y, z, v, \tau ; \bar{V}) \simeq \tilde{D}-\tilde{D}(0, z, v, \tau ; \bar{V})+\frac{1}{2} y^{2} \tilde{D}^{\prime \prime}(z, v, \tau ; \bar{V}),
$$

where

$$
\tilde{\mathrm{D}}^{\prime \prime}(\mathrm{z}, \mathrm{v}, \tau ; \overline{\mathrm{V}}) \equiv-\left.\frac{\partial^{2}}{\partial \mathrm{y}^{2}} \tilde{\mathrm{D}}(\mathrm{y}, \mathrm{z}, \mathrm{v}, \tau ; \overline{\mathrm{V}})\right|_{\mathrm{y}=0},
$$

is the same (positive) object introduced in Sec. 4 [see Eq. (36)]. Its $\mathrm{z}$ dependence serves to properly define the time-dependent diffusion coefficient taken along the (relative) particle orbit. Because the coherent parts of Eqs. (66) and (65) are identical, $\tilde{D}_{-}(y=0)$ vanishes for small $z$. This says physically that the particle cannot diffuse azimuthally when pinned to its initial line $\left(\rho_{i}^{j}\right.$ is held fixed during the relative motion). It also proves that the conventional resonance broadening term which would arise from just the $\partial_{\rho} \tilde{D} \partial_{\rho}$ term, is inappropriate for small relative separations, the regime of exponential divergence. [The discussion following $\mathrm{Eq}$. (25) can now be recalled.]

The solution of Eq. (66) with. $\tilde{D}_{-} \simeq(1 / 2) \mathrm{Y}^{2} \mathrm{D}^{\prime \prime}$ is complicated. A useful approximation is to assume that $K$ is Gaussian, with moments determined frnm Fif. (66). This appruxillation 15 correct at short times, and also at long times when $\tilde{D}\left(\rho_{\sim}\right)$ vanishes and $K$ factors into $R F$, with $R$ given by Eq. (21). Here, we simply extrapolate the short time solution. Since we need $\mathrm{K}$ only for 
$\tau<\tau_{\delta}$, this approximation should be in error by unimportant factors inside the logarithm involved in $\tau_{\delta}$.

Let us define

$$
\overline{\mathrm{K}}(\underset{\sim}{\rho}, \mathrm{V}, \tau ; \overline{\mathrm{V}}) \equiv \hat{\mathrm{K}}\left(\underset{\sim}{l}, \underset{\sim}{\rho}, \mathrm{V}, \tau ; \mathrm{V}^{\prime}\right) / \mathrm{F}(\underset{\sim}{1}) \vdots \quad
$$

From Eq. (66) or more generally from Eq. (57),

$$
\int \underset{\sim}{d \rho} \mathrm{dV} \overline{\mathrm{K}}=1 \quad(\tau>0),
$$

so we can interpret $\overline{\mathrm{K}}$ as a probability density for relative motion. Moments of $\overline{\mathrm{K}}$ are defined as $\langle\mathrm{A}(\underset{\sim}{\rho}, \mathrm{V})\rangle \equiv \int \mathrm{d} \underset{\sim}{\rho} \mathrm{dV} \mathrm{A}(\underset{\sim}{\rho}, \mathrm{V}) \overline{\mathrm{K}}$. We find from Eq. (66) that

$$
\begin{aligned}
& \langle y\rangle=-y_{1}, \\
& \langle\rho\rangle=-\rho_{1} .
\end{aligned}
$$

These results can also be written as $\left\langle y_{2}\right\rangle=\left\langle p_{2}\right\rangle=0$, which is a statement of the symmetric azimuthal and radial motions of particles which pass through $\mathrm{y}_{2}=0, \rho_{2} \doteq 0$ at $\mathrm{z}_{2}=0, \tau=0$. For definiteness, let us now consider the collisionless limit in detail. The second moments $y \equiv\left\langle y^{2}\right\rangle, M=\langle y \rho\rangle, R=\left\langle\rho^{2}\right\rangle$ then obey approximately 


$$
\begin{aligned}
& \frac{\partial}{\partial \tau} y-\frac{2 V^{\prime}}{L_{s}} \delta M-2 D_{\perp}=y_{1}^{2} \delta(\tau), \\
& \frac{\partial}{\partial \tau} \delta M-\frac{V^{\prime}}{L_{s}} \delta R=0, \\
& \frac{\partial}{\partial \tau} \delta R-\overline{\mathrm{k}}_{\theta}^{2} D^{\prime \prime}\left(V^{\prime}\right) y=0,
\end{aligned}
$$

where $\delta M \equiv H(\tau)(M-\langle y\rangle\langle\rho\rangle), \delta R \equiv H(\tau)\left(R-\langle\rho\rangle^{2}\right)$, and

$$
D^{\prime \prime}\left(V^{\prime}\right) \equiv \int d V \cdot D^{\prime \prime}\left(V ; V^{\prime}\right)
$$

Equations (69) reduce to

$$
\ddot{y} \dot{(\tau)}=\left(\frac{2 V^{\prime}}{L_{K}}\right)^{3} y,
$$

with initial values $y(0)=y_{I}^{2}, \dot{y}(0)=2 D_{\perp}, \ddot{y}(0)=0$; the asymptotic solution is

$$
\delta y=\frac{l}{3} \bar{k}_{\theta}^{-2}\left[\left(\bar{k}_{\theta} y\right)^{2}+\tau_{\delta} / \bar{\tau}_{\perp}\right] \exp \left(2 \tau / \tau_{\delta}\right)
$$

Similarly, it can be shown that 


$$
\begin{aligned}
& \langle V\rangle=\left\langle V\left(\tau \mid V^{\prime}\right)\right\rangle \\
& \langle z\rangle=-z_{1}+\left\langle z\left(\tau \mid V^{\prime}\right) .\right\rangle \\
& \left\langle\delta V^{2}\right\rangle=\sigma_{V}^{2}(\tau), \\
& \left\langle\delta V \delta z>=r(\tau) \sigma_{V}(\tau) \sigma_{z}(\tau),\right. \\
& \left.: \delta z^{2}\right\rangle=\sigma_{z}^{2}(\tau),
\end{aligned}
$$

that the cross moments $\langle\delta V \delta \rho\rangle$ and $\langle\delta z \delta \rho\rangle$ vanish, and that $\langle\delta V \delta y\rangle$ and $\langle\delta z \delta y\rangle$ vanish when third and higher cumulants are neglected. If we take conjugate Fourier transform pairs to be $[(\mathrm{z}, \mathrm{k}),(\mathrm{Y}, \lambda),(\rho, l),(\mathrm{V}, \mu)\} \equiv\{(\underset{\sim}{\rho}, \underset{\sim}{\mathrm{k}}),(\mathrm{V}, \mu)\} \equiv\{(\underset{\sim}{\mathrm{x}}, \underset{\sim}{\mathrm{K}})\}$, then the Gaussian approximation

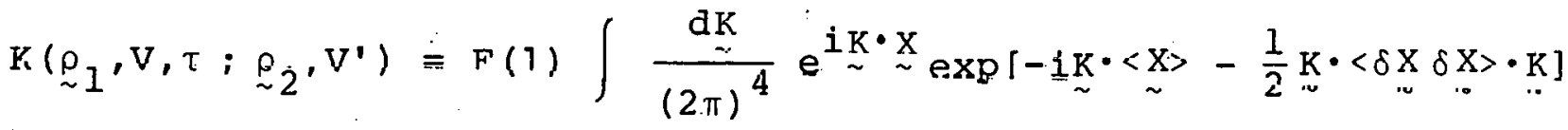

reduces to

$$
\begin{aligned}
K(1,2)= & : F(1) \int \frac{d \lambda d l}{(2 \pi)^{2}} e^{i \lambda Y_{2}+i l \rho} 2, P\left(V, z_{2}, \tau ; V^{\prime}\right) \\
& \times \exp \left[\frac{1}{2}\left[\lambda^{2} \delta !(\tau)+2 \lambda \ell \delta M(\tau)+\ell^{2} \delta R(\tau)\right]\right\}
\end{aligned}
$$

To define the diffusion coefficient, we consider the spatial Fourier transform of Eq. (65): 


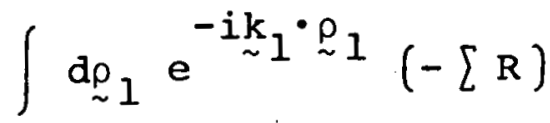

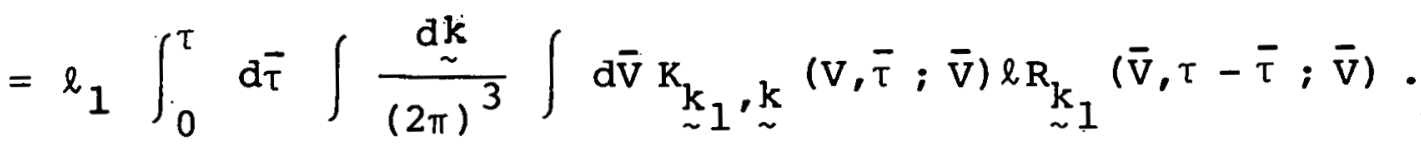

The kinetic equation in the hydrodynamic limit $\left(k_{1}, \lambda_{1}\right)=0$, $\ell_{1} \rightarrow 0$ becomes

$$
\partial_{\tau} R_{\ell_{1}}-C R_{\ell_{1}}-\sum_{\ell_{1}} R_{\ell_{1}}=\delta\left(V-V^{\prime}\right) \delta(\tau)
$$

This admits a factored solution of the form

$$
R_{\ell}\left(V, \tau ; V^{\prime}\right)=H(\tau) \hat{R}\left(V, \tau ; V^{\prime}\right) n_{\ell}(\tau),
$$

where

$$
\mathrm{n}_{\ell}(\tau) \equiv \int \mathrm{dV} \mathrm{R}_{\ell}\left(\mathrm{V} ; \tau ; \mathrm{V}^{\prime}\right)
$$

and

$$
\left(\partial_{\tau}-C\right) \hat{R}=0, \hat{R}\left(V, 0 ; V^{\prime}\right)=\delta\left(V-V^{\prime}\right)
$$

Integrating Eq. (73) over velocity, we find

$$
\frac{\partial}{\partial \tau} n_{\ell_{1}}(\tau)+\left(-\int d v d \bar{v} \int_{0}^{\tau} d \bar{\tau} \sum_{\ell_{1}}(v, \bar{\tau} ; \bar{v}) \hat{R}\left(V, \tau ; V^{\prime}\right)\right) n_{\ell_{1}}(\tau)=0,
$$

where we have made the Markovian approximation $R(\tau-\bar{\tau}) \simeq R(\tau)$, appropriate for $\tau>\tau_{\delta}$. For $\nu \tau>1, R$ is a Maxwellian $\phi_{M}(V)$ normalized to one. Inserting Eq. (71) into Eq. (72) and neglecting 
the radial dependence of $F$, we find that the bracketed term in Eq. (74) reduces to $\ell_{1}^{2} \mathrm{D}$, where

$$
\begin{aligned}
D=\int_{0}^{\tau} d \bar{\tau} \int \frac{d k d \lambda}{(2 \pi)^{2}} \int & d V d \bar{V} F_{-\lambda,-k} \exp \left[-\frac{1}{2} \lambda^{2} \delta y(\bar{\tau})\right] \\
& \quad \times V_{k}(V, \bar{\tau} ; \bar{V}) \bar{V} \phi_{M}(\bar{V})
\end{aligned}
$$

Since $F_{-\lambda,-k}=F_{\lambda, k}$ by reality and since

$$
\int_{\Delta \mathrm{k}_{\|}} \frac{\mathrm{dk}}{2 \pi} \mathrm{P}_{\mathrm{k}}=\int \mathrm{dz} \delta(\mathrm{z} ; \Delta \mathrm{k} \| \mathrm{P}(\mathrm{z})
$$

we see that Eq. (75) is identical to the average of Eq. (26) over a thermal distribution of velocities. The collisional limit can be treated similarly.

We have given only a very approximate solution of the kinetic equation (63). Further work is clearly desirable. However, we do see that the content of this equation, unlike the quasilinear equation or the DIA, contains the physics in which we are interested -- namely, the decorrelation effect due to line divergence. This fundamental observation is one of the principal results of this paper. 


\section{Discussion}

We have developed a theory of electron transport in stochastic magnetic fields by employing the techniques of kinetic theory. Our approach permits a systematic study of parameter space, predicts the characteristic lengths of the stochastic fields, and is capable, at least in principle, of producing numerical coefficients.

In the body of the paper, we considered the limit $\mathrm{L}_{\mathrm{O}} / \mathrm{L}_{\mathrm{K}}<1$. This is a reasonable limit, since it is the regime passed through first as the level of unstable magnetic fluctuations rises through the stochasticity threshold (at least if the modal density and localization remain reasonably constant). It is interesting to estimate $\mathrm{L}_{O} / \mathrm{L}_{K}$. for the parameters discussed by RR. If we take $\delta \sim 10^{-4}$ and $\Delta r \sim \rho_{i}, a / \rho_{i} \sim 10^{-2}$, we find from Eq. (23) that

$$
L_{O} / I_{K} \simeq 10^{-4 / 3}<1 / 10
$$

If the fluctuations were localized less strongly in radius, $\mathrm{k}_{\theta} \Delta \mathrm{r}>1, \mathrm{~L}_{\mathrm{O}} / \mathrm{L}_{\mathrm{K}}$ would be even smaller.

One can infer scaling laws for the opposite limit $L_{K} / L_{0}<1$. Because of Eq. (22) the relvant correlation length is now $L_{K}^{(r)}$ $\equiv L_{r} \equiv\left(k_{r}^{2} D_{m}\right)^{-1}$. For $L_{o}<\ldots l$ we are then in regime $(32 \mathrm{a})$, so that

$$
D \sim V_{0} D_{m}^{(0)}\left(I_{r} / I_{0}\right)=V_{o} D_{m}
$$


where $D_{m}$ is the renormalized diffuision coefficient:

$$
\mathrm{D}_{\mathrm{m}} \sim\left[\mathrm{D}_{\mathrm{m}}^{(\mathrm{O})} / \mathrm{L}_{\mathrm{o}}\right]^{1 / 2} \Delta \mathrm{r}
$$

We did not treat in detail the self-consistent próblem. Because our description is kinetic, however, our formalism generalizes readily. In terms of the discussion of sec. 5, the necessary additional terms describe the averaged effects of small perturbations of the microscopic fields, e.g., < $\left.\delta b / \delta \eta_{-}\right\rangle$. The role of these terms is well-known (Krommes 197:7; Thompson \& Krommes 1977; Espedal \& Dubois 1977); they describe the selfconsistent Vlasov fields, dielectric properties of the background, and mode-coupling. These terms must be studied in the context of a specific instability. Such work is well underway (Kleva 1978).

We have omitted from the analysis magnetic drifts in the unperturbed tokamak field. Preliminary estimates indicate that such drifts have relatively small effects on the stochastic $D=\int d \tau<V_{\|} b V_{\|} b>$ unless the drift excursions are larger than the radial correlation length. Also contributing to the radial diffusion is the arift term $D_{d} \equiv s d \tau v_{d}^{2}\langle\sin [\theta(\tau)] \sin [\theta(0)]\rangle$, where $v_{d}$ is the combined gradient and curvature drift. In the absence of radial perturbations, this term gives the usual neoclassical diffusion coefficient. For $b \neq 0$, preliminary estimates suggest that in the collisionless limit the neoclassical result is unchanged unless $\omega_{b} \tau_{\delta}<1$, in which case $D_{d} \sim v_{d}^{2} \tau_{\delta}$. Here $\omega_{b}$ is the usual magnetic bounce frequency: $\omega_{b}=V / R q$. Since one 
finds $\omega_{b} \tau_{\delta} \sim\left(L_{K} / L_{s}\right)$ for reasonable tokamak parameters, this regime requires a strong level of turbulence.

We wish to comment on the renormalization technique we employed in Sec. 5. The MSR procedure is distinct from many others (Dupree 1966; Weinstock 1969; Birmingham \& Bornatici 1971; Rudakov \& Tsytovich 1971; Krommes \& Oberman 1976) in that the equations are couched from the very beginning in terms of observables (fewpoint averages); fluctuating quantities never appear, nor do highorder cumulants. It is, of course, the observables which can be small; fluctuations may be singular.

The MSR description is in terms of Eulerian correlation functions. For extremely large nonlinearity, this description is sometimes deficient; for example, random Galilean invariance is not preserved for fluids at high Reynolds numbers (Kraichnan 1964b). Kraichnan has developed a renormalized description [the Lagrangian History DIA (Kraichnan 1965)] which, though not completely systematic, overcomes this problem. Though we have not examined this theory in detail for the problem of stochastic transport, we belleve that we could recover the principle results of this paper from the LHDIA.

In conclusion, though our practical concern has been with transport in stochastic magnetic fields, the kinetic approach we have initiated is clearly more general. The techniques should prove useful in a variety of classical situations involving stochastic instability. In plasmas, one is immediately reminded of stochastic heating, as well as various aspects of containment in 
mirror machines. The quantitative kinetics of these situations are by no means fully explored; their further study represents a fascinating area for future work.

We are very grateful for stimulating conversations with M. Rosenbluth, A. Rechester, and G. Laval. We are especially indebted to Gary Smith, who aroused the initial interests of one of us (JAK) in stochasticity and who has participated with us in many enjoyable and informative discussions.

This work was jointly supported by U. S. Air Force office of Scientific Research Contract No. F 44620-75-C-0037 and by U. S. Department of Energy Contract No. EY-76-C-02-3073. 
Appendix A: Reduction of Integrals for Parallel Transport Problem

Consider the integral

$$
d(t) \equiv 2 \int_{0}^{t} d \tau \int d V d z P(z, V, \tau) V C_{\|}(z)
$$

which describes the dynamics of parallel transport. The $\mathrm{V}$ integration is straightforward:

$$
\int \operatorname{dVVP}(z, V, \tau)=\left[(\ln \sigma)^{\bullet} \delta z+\langle V>] P(\delta z)\right.
$$

where we write $\sigma \equiv \sigma_{\mathrm{z}}$, note $\dot{\sigma}=\mathrm{r} \sigma_{\mathrm{v}}$, and define

$$
P(z) \equiv\left(2 n \sigma^{2}\right)^{-1 / 2} \exp \left(-z^{2} / 2 \sigma^{2}\right)
$$

If we introduce the Fourier coefficients $C_{k}$ of $C_{\|}^{(z)}$, change variables to $\delta z$ in definition (A1), and use Eq. (A2), we find

$$
d(t)=2 \int \frac{d k}{2 \pi} c_{k} \int_{0}^{t} d \tau \exp \left(i k<z>-\frac{1}{2} k^{2} \sigma^{2}\right) \frac{d}{d \cdot \tau}\left(\frac{1}{2} i k \sigma^{2}+<z>\right) \text {. }
$$

The $\tau$ integral is the perfect derivative of the exponential, divided by ik :

$$
d(t)=2 \int \frac{d k}{2 \pi i k} C_{k}\left[\exp \left(i k<z>-\frac{1}{2} k^{2} \sigma^{2}\right)-1\right] .
$$


We can express (A4) as an integral over average position:

$$
\left.d(t)=2 \int_{0}^{<z(t)>} d \zeta<C_{\|}(z)\right\rangle_{\zeta},
$$

where

$$
\left\langle q_{\|}(z)\right\rangle_{\zeta} \equiv \int d(\delta z) C_{\|}(\zeta+\delta z) P(\delta z, t), \quad
$$

and we used the convolution theorem and the boundary condition $d(0)=0$. We consider two examples.

Example 1: $C_{\|}^{(z)}=\delta(z)$.

This example corresponds to the case of a completely nonlocalized spectrum, $L_{0}=0$. We find $\left\langle C_{\|}(z)\right\rangle_{\zeta}=P(\zeta, t)$ and

$$
a(t)=\operatorname{erf}[<z(t)>/ \sqrt{2} \sigma(t)]
$$

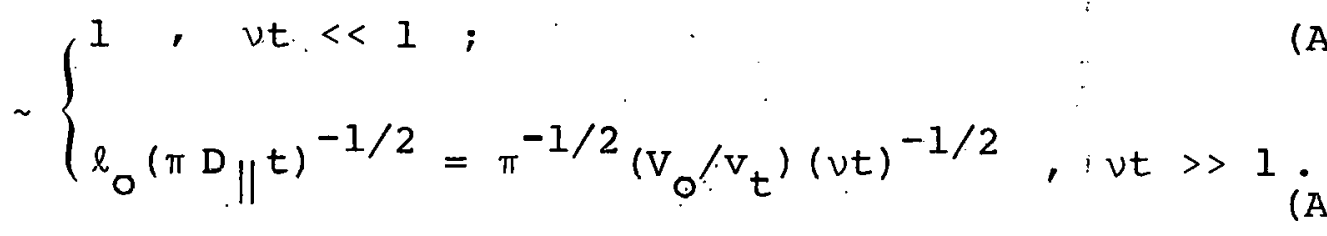

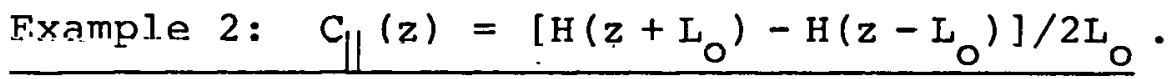

This function has the same qualitative features as $\delta\left(\mathrm{z} ; \Delta \mathrm{k}_{1}\right)$. Straightforward integration leads to 


$$
\begin{aligned}
d(t)=\left(\frac{\sqrt{2} \sigma}{2 L_{0}}\right)\left\{\left[\operatorname{erfc}_{1}\left(\frac{\langle z\rangle+L_{0}}{\sqrt{2} \sigma}\right)-\operatorname{erfc}_{1}\left(\frac{\langle z\rangle-L_{0}}{\sqrt{2} \sigma}\right)\right]\right. \\
\left.\left.\quad-\operatorname{erfc}_{1}\left(L_{0} / \sqrt{2} \sigma\right)-\operatorname{erfc}_{1}\left(-L_{0} / \sqrt{2} \sigma\right)\right]\right\}, \text { (A7) }
\end{aligned}
$$

where $\operatorname{erfc}_{1}(z) \equiv \int_{z}^{\infty} \operatorname{duerfc}(u)$. For $\langle z\rangle>L_{0}, E q \cdot$ (A7) reduces to Eq. (A5). For the short time limit $\langle z\rangle\left\langle\mathrm{L}_{0}\right.$, which need not imply $v t<1$, we have

$$
\begin{aligned}
d(t) & \simeq \frac{\langle z\rangle}{L_{0}} \operatorname{erf}\left(L_{0} / \sqrt{2} \sigma\right) \\
& \sim\left\{\begin{array}{l}
\left\langle z(t)>/ L_{0}, \sigma<L_{0} ;\right. \\
(2 \pi)^{-1 / 2}<z(t)>/ \sigma(t),
\end{array},\right.
\end{aligned}
$$

Furthermore,

$$
\left\langle z(t)>/ L_{0} \sim \begin{cases}V_{0} t / L_{0}, & \sigma<\left\langle z<L_{0} ; v t<1\right. \\ l_{0} / L_{0}, & z z\rangle<\sigma<L_{0} ; v t>1 .\end{cases}\right.
$$

Because of the restriction $\langle z\rangle\left\langle L_{0}\right.$, Eq. (A9b) reduces to Eq. $(A 6 b)$.

We graph these cases in Figs. I- 3 along with their derivative

$$
\begin{aligned}
d^{\prime}(t) & =\left(\frac{\partial}{\partial t}+\langle V\rangle \frac{\partial}{\partial\langle z\rangle}\right) d[\sigma(t),\langle z\rangle] \\
& =(\ln \sigma)^{\circ} d(t)+\left[\langle V\rangle-(\ln \sigma)^{\bullet}\langle z\rangle\right] \partial d / \partial\langle z\rangle,
\end{aligned}
$$


where

$$
\frac{\partial d}{\partial\langle z\rangle}=\frac{1}{2 L_{0}}\left[\operatorname{erf}\left(\frac{\left\langle z>+L_{0}\right.}{\sqrt{2} \sigma}\right)-\operatorname{erf}\left(\frac{<z>-L_{0}}{\sqrt{2} \sigma}\right)\right] \text {. }
$$


Appendix B: Aspects of Renormalized Perturbation Theory

We give here a cursory discussion of the first few terms of the renormalized perturbation theory of stochastic differential equations of the form Eqs. $(53,54)$. Let us define the unperturbed, coherent response function $\mathrm{R}^{(0)}\left(1,1^{\prime}\right)$ by the solution of

$$
\left(\frac{\partial}{\partial t_{1}}+i L_{1}\right) R^{(\odot)}\left(1,1^{\prime}\right)=\delta\left(1-1^{\prime}\right)
$$

Equation (53) can then be rewritten as the integral equation

$$
\hat{R}\left(1,1^{\prime}\right)=R^{(0)}\left(1,1^{\prime}\right)-R^{(0)}(1, \bar{I}) i \omega(\bar{I}) \hat{R}\left(\bar{I}, 1^{\prime}\right) \text {. }
$$

Suppose that the stochastically nonlinear term $\propto \omega$ were small. [It is not small, in general, especially near zeros of $\partial_{t}+i L$ (resonances)]. The iterative solution of (BI) then yields in $\mathrm{n}-\mathrm{th}$ order

$$
R^{(n)}=\left\langle\hat{R}^{(n)}\right\rangle=\left\langle\left[-R^{(0)} i \omega\right]^{n}\right\rangle R^{(0)} .
$$

We have defined the averaging operator so that $i(1) \propto b$ is Gaussian; all odd moments $\langle i \omega(1) \ldots i \omega($ odd) $\rangle$ then vanish. (This procedure is possible in the present problem where $b$ is specified; it is not, in general, possible in the self-consistent problem where the nonlinear coupling of the finite- $\beta$ modes arive in nonvanishing cumulants of all orders even if the cumulants were initially 
Gaussian.) Thus, e.g.,

$$
R^{(2)}=R^{(0)}<(-i \omega) R^{(0)}(-i \omega)>R^{(0)}
$$

(Fig. 8a),

$$
\begin{aligned}
R^{(4)}=R^{(0)}<(-i \omega) R^{(0)}(-i \omega)>R^{(0)}<(-i \omega) R^{(0)}(-i \omega)>R & (0) \\
& +(\text { two more terms) }
\end{aligned}
$$

(Fig. 8b), where, in Fig. 8, the curly lines denote correlations, or averaging, dots denote the bare interaction vertex - iw, and the thin horizontal lines denote the unperturbed response function $R^{(0)}$. The object $Q^{(0)}$ defined by $\left\langle i \omega R^{(0)} i \omega\right\rangle$ is the non-Markovian version of the quasilinear operator: for arbitrary $\mathrm{H}$,

$$
\left.\mathrm{Q}^{(0)} \mathrm{H} \equiv \int_{0}^{t} \mathrm{~d} \tau \frac{\partial}{\partial r} \mathrm{VR}^{(0)}(\underset{\sim}{\underline{1}}, \tau ; \overline{\underline{1}}) \overline{\mathrm{V}}<\underset{\sim}{i} \omega \underset{\sim}{(1)} \underset{\sim}{i} \omega \underset{\sim}{\overline{1}}\right)>\frac{\partial}{\partial \dot{\bar{r}}} \mathrm{H}(\underset{\sim}{\overline{1}}, t-\tau),
$$

though note that the equation for $\mathrm{R}$ correct through second order,

$$
\mathrm{R}^{(0)^{-1}} \mathrm{R}=\mathrm{Q}^{(0)} \mathrm{R}^{(0)}+1
$$

is not a true quasilinear equation because of the presence of $R^{(0)}$ rather than $R$ following $Q^{(0)}$ : However, it is not hard to see and has been discussed in many places (Tetreault 1976), that the higher order disconnected terms (with no crossed lines), 
e.g., the first two terms of Fig. (8b) or Fig. (9a), sum to replace $R^{(0)}$ by $R$ everywhere on the right hand side of Eq. (B3), giving the renormalized quasilinear equation

$$
\left.\mathrm{R}^{(0)}\right)^{-1} \mathrm{R}=\mathrm{QR}+1
$$

More generally, the sum of all disconnected diagrams (including crossed lines) yields the mass operator or turbulent collision operator $\Sigma$ and the Dyson equation (Frisch 1968)

$$
\mathrm{R}^{(0)} \mathrm{R}-\sum \mathrm{R}=1
$$

Terms involving crossed lines describe intrinsic two-body correlations. These are defined as those parts of the joint probability $\mathrm{K}\left(\begin{array}{l}1,2,3^{3,4} \\ 0\end{array}\right)$, of the field ("0") at 1 and a particle ("+") at 2 , given the field at 3 and a perturbation ("-") in the particle probability at 4 , which do not factor into the product $<\mathrm{b}(1) \mathrm{b}(3)>\mathrm{R}(2,4)$ of the singlet probabilities. To see this more clearly, consider the last term of Fiq. (8b) and a.1 similar higher order terms such as Fig. (9b), each connected factor of which contains at most one crossed line. These crossed terms sum to

$$
R\left(I, 1^{\prime}\right)=R^{(0)}(1,2) v_{2} \frac{\partial}{\partial r_{2}} \tilde{K}\left(2^{\prime}, 2 ; \bar{l}, \bar{l}\right) \mid 2^{\prime}=2^{V_{\bar{l}}} \frac{\partial}{\partial r_{\bar{l}}} R\left(\bar{l}, 1^{\prime}\right),
$$

where

$$
\begin{aligned}
\tilde{\mathrm{K}}(1,2 ; 3,4) & =\mathrm{F}(1,3) \mathrm{R}(2,4) \\
& +\mathrm{R}(2, \overline{2})\left(\mathrm{V}_{2}-\frac{\partial}{\partial \mathrm{r}_{\overline{2}}} \mathrm{R}(\overline{2}, \overline{4}) \mathrm{F}(1, \overline{4}) \mathrm{V}_{\overline{4}} \frac{\partial}{\partial r_{\overline{4}}}\right) \mathrm{R}(\overline{4}, 4) \mathrm{F}(\overline{2}, 3) .
\end{aligned}
$$


The last term arises from the crossed graphs and is clearly nonfactorable [note the term $F(1, \overline{4}) \mathrm{R}(\overline{4}, 4)]$. The form of (B4b) strongly suggests that $\tilde{\mathrm{K}}$ represents the first two terms of an expansion of $k$ in powers of $\omega^{2}$. It does not elucidate the nature of the neglected terms. The technique of Martin, Siggia, and Rose (1973), which we discuss in the main text, permits this and allows a systematic treatment of all orders of renormalization. 
ARNOL'D, V.I. 1963 Russian Math. Surveys 18, 9; 18, 85. BALESCU, R. 1975 Equilibrium and Nonequilibrium Statistical

Mechanics. N.Y.: Wiley.

BIRMINGHAM, T. \& BORNATICI, M. 1971 Phys. Fluids 14, 2239.

BOURRET, R.C. 1962 Nuovo Cimento 26, 1 .

CALLEN, J.D. 1977 Phys. Rev. 39, 1540.

CHANDRASEKHAR, S. 1943 Rev. Mod. Phys. 15, 1. In Selected

Papers on Noise and Stochastic Processes (ed. N. Wax), p. 3 .

Dover: New York (1954).

CHEN, L., RUTHERFORD, P.H. \& TANG, W.M. 1977 Phys. Rev. Lett. 39, 460 .

CHIRIKOV, B.V. 1969 "Research Concerning the Theory of Nonlinear Resonance and Stochasticity" (Rep. 267, Nucl. Phys. Inst. of Siberian Sect. USSR Acad. Sci., Novosibirsk), trans. CERN (Geneva, 1971), unpublished.

CHIRIKOV, B.V. $1977 \Lambda$ Universal Instability of Many-Dimensional Oscillator Systems (Institute of Nuclear Physics, Novosibirsk) in preparation for Rev. Mod. Phys.

DeDOMINICIS, C. \& MARTIN, P.C. 1964 J. Math. Phys. $\underline{5}, 14$. DEKER, U. \& HAAKE, F. 1975 Phys. Rev. All, 2043. DIIPRFE; T.H. 1966 Fhys. Flulds $\underline{\underline{g}}, 1773$. DUPREE, T.H. 1972 Phys. Fluids 15, 334. ESPEDAL, M. \& DUBOIS, D.F. 1970 Bull. Am. Phys. Soc. $22,1149$. FORD, J. 1975 In Fundamental Problems in Statistical Mechanics (ed. E.D.C. Cohen), Vol. 3, p. 215. North-Holland Publ. Co.: Amsterdam. 
FRISCH, U. 1968 In Probabilistic Methods in Applied Mathematics

(ed. A.T. Bharucha-Reid) p. 75. Academic Press, N.Y. HINTON, F.L. \& HAZELTINE, R.D. 1976 Rev. Mod. Phys. 48, 239. JOKIPII, J.R. \& PARKER, E.N. 1969 Ap. J. 155, 777.

KADANOFF, L.P. \& MARTIN, P.C. 1963 Ann. Phys. 24, 419. KLEVA, R.G. 1978 Ph.D. thesis, Princeton University (in preparation). KOLMOGOROV, A.N. 1954 Dokl. Akad. Nauk SSSR 98, 527 (trans.

LA-TR-71-67 Los Alamos Scientific Lab., Los Alamos, New Mexico). KRAICHNAN, R.H. 1958 Phys. Rev. 109; 1407. KRAICHNAN, R.H. 1961 J. Math. Phys. 2., 124. KRAICHNAN, R.H. 1964 a Phys. Fluids 7, 142. KRAICHNAN, R.H. 1964b Phys. Fluids ㄱ, 1723. KRAICHNAN, R.H. 1965 Phys. Fluids $\underline{8}, 575$. KROMMES, J.A. 1977 "Turbulence, Clumps, and the Bethe-Salpeter

Equation" Proc. Third Kiev-Theory Conf. (Trieste) to be published. KROMMES, J.A. \& OBERMAN, C. 1976 J. Plasma Phys. 16, 193. KROMMES, J.A. \& SMITH, G.R. 1976 Bull. Am. Phys. SOC. $21,11 b 2$. MARTIN, P.C., SIGGIA, E.P. \& ROSE, H.A. 1973 Phys. Rev. A8, 423. MOSER, J. 1973 In Stable and Random Motions in Dynamical Systems

Princeton University Press.

NISHIJIMA, K. 1969 In Fields and Particles Chap. 7. N.Y.: Benjamin. ORSZAG, S.A. 1977 In Fluid Dynamics (ed. R. Balian \& J.-L. Peube),

p. 235. Paris: Gordon \& Breach Science Publishers. PHYTHIAN, R. 1975 J. Phys. A8, 1423. RECHESTER, A.B. \& ROSENBLUTH, M.N. 1978 Phys. Rev. Lett. 40,38 . RECHESTER, A.B. \& STIX, T.H. 1976 PhYs. Rev. Lett. 36, 587. 
ROSE, H.A. 1974 Ph.D. thesis, "Aspects of the Statistical Dynamics of Classical Systems" (Harvard University) unpublished. ROSENBLUTH, M.N., SAgDEEV, R.Z., TAYLOR, J.B. \& ZASLAVSKII, G.M. 1966 Nucl. Fusion 6 , 297. RUDAKOV, L.I. \& TSYTOVICH, V.N. 1971 Plasma Phys. 13, 213. SMITH, G.R. \& KAUFMAN, A.N. 1975 Phys. Rev. Lett. 34, 1613. SMITH, G.R. \& KAUFMAN, A.N. 1976 "Diffusion due to a Single Wave in a Magnetized Plasma" Proc. of the Nobel Symposium on Nonlinear Effects in Plasmas (Sweden) to be published. SMITH, G.R. 1977a Phys. Rev. Lett. 38, 970. SMITH, G.R. 1977b "Numerical study of Particle Motion in Two Waves" Proc. Int. Conf. on Stochastic Behavior in Classical and Quantum Hamiltonian Systems (Como, Italy) to be published. SMITH, G.R. \& KAUFMAN, A.N. 1978 Phys. Fluids (submitted). STIX, T.H. 1973 Phys. Rev. Lett. $\underline{30}, 833$. STIX, T.H. 1976 "Aspects of Stochastic Heating" (unpublished). TAYLOR, G.I. 1921 Proc. Lond. Math. Soc. A20, 196.

TETREAULT, D.J. 1976 Ph.D. thesis, "Renormalization of the waveParticle Resonance in Turbulent Plasma" (MIT, Cambridge) unpublished.

TFR GROUP 1977 In Proceedings of the 6th Int. Conf. in Plasma Phys. and Controlled Nuclear Fusion Research (IAEA, Vienna), Vol. I, p. 35.

THOMPSON, H.R. \& KROMMES, J.A. 1977 Bull. Am. Phys. Soc. 22, 1150. VAN KAMPEN, N.G. 1976 Phys. Rep. 24C, 172. WALKER, G.H. \& FORD, J. 1969 PhYS. Rev. 188, 416. 
WEINSTOCK, J. 1969 Phys. Fluids 12, 1045.

ZASLAVSKII, G.M. \& CHIRIKOV, B.V. 1971 Usp. Fiz Nauk. 105, 3 [trans. Sov. Phys. Uspekhi 14, 549 (1972)]. 


\section{Figure Captions}

Figure 1. Time dependence of the normalized "diffusion" coefficient $d(t)$ for parallel transport. The solid line represents $d(t)$ for Example 2, Appendix $A$, with $L_{0} / \ell=0.1$. The dashed line corresponds to $d(t)$ for Example 1, Appendix A $\left(L_{0}=0\right)$. The long-short dashed line is the derivative of the solid curve (axis to right).

Figure 2. Same as Figure 1, except that $\mathrm{L}_{\mathrm{o}} / \ell=1.0$.

Figure 3. Same as Figure 1, except that $L_{0} / l=5.0$.

Figure 4. (a) The Dyson equation. The thick line denotes the renormalized correlation matrix G, the thin line denotes the bare matrix $G_{0} \cdots\left(\partial t-U_{2}\right)^{-l}$. Dashed lines indicate simple multiplication. The function $\Phi$ is the state vector, $\Sigma$ is the mass operator. (b) The mass operator. Small dot denotes the bare vertex $\gamma, K$ is the two-body scattering matrix. (c) Exact relation between the renormalized vertex (large dot) and the scattering matrix $\mathrm{K}$. (d) Alternate representation of $\Sigma$ which follows from (c) and (b). (e) The Direct Interaction Approximation $\Gamma \simeq \gamma$.

Fiqure 5. (a) The Bethe-Salpeter Equation. 'l'he interaction matrix $I$ is defined in Eq. (58). (b) Lowest nonvanishing approximation to I in the renormalized skewness expansion. (c) Generalized diffusion approximation $I \simeq \gamma G \gamma$ to the Bethe-Salpeter Equation. 
Figure 6. Diagrammatic representations of (a) the two-point correlation $F$ of the magnetic field; (b) the particle response function $R$.

Figure 7. Explicit form of the diffusion approximation to the Bethe-salpeter Equation for the joint probability ' $\mathrm{K}(0+; 0-)$ of the particle and field.

Figure 8. Perturbation theory approximations for the response function $R$. Straight line denotes the bare propagator. Dot denotes the bare vertex and random coefficient. Wiggly line denotes averaging. (a) second-order $R ;$ (b) fourth-order $R$.

Figure 9. Examples of tenth-order contributions to R. (a) A term which renormalizes the propagator. (b) A term which renormalizes the vertex. 


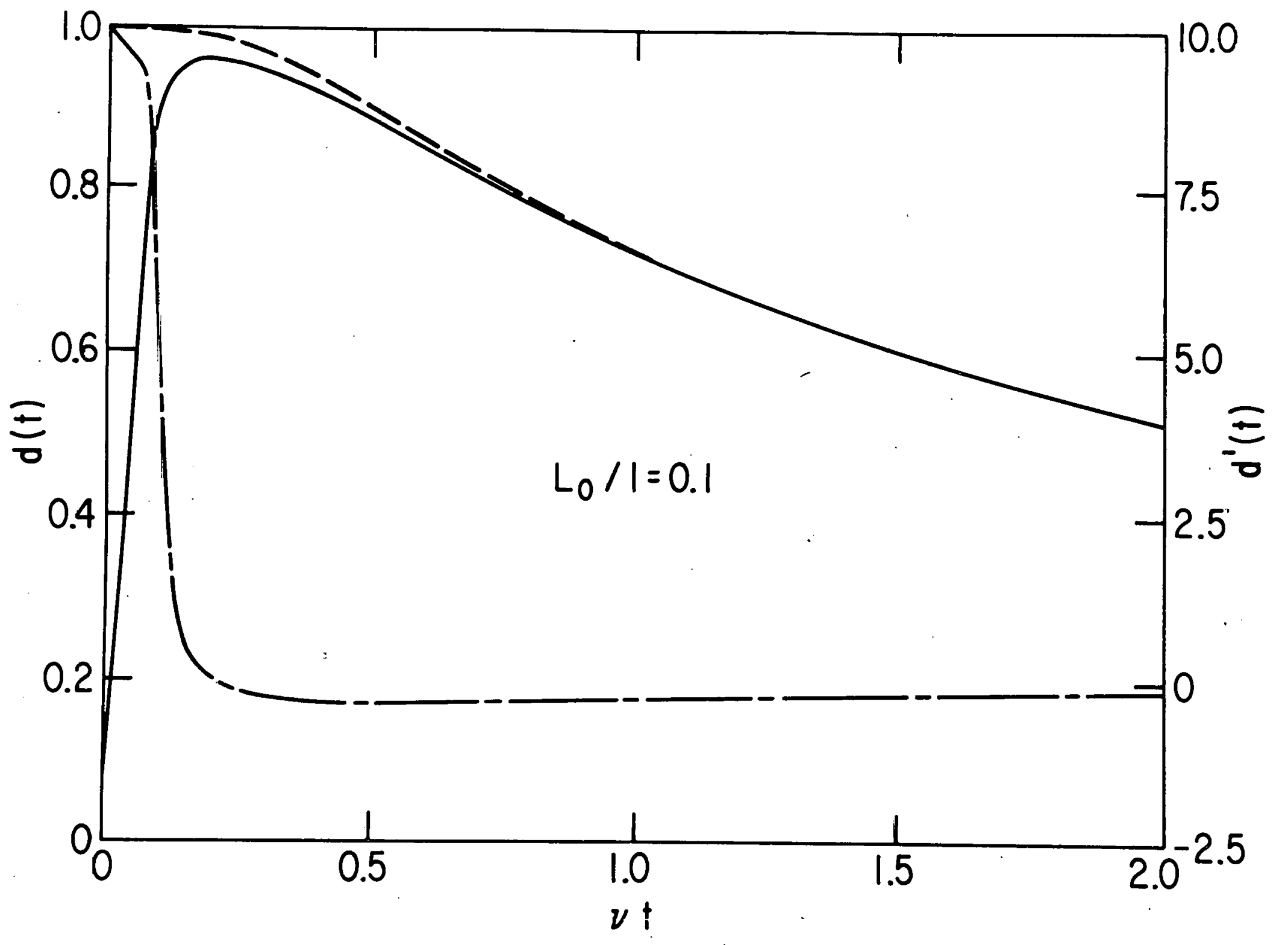

Fig. 1. 782079 


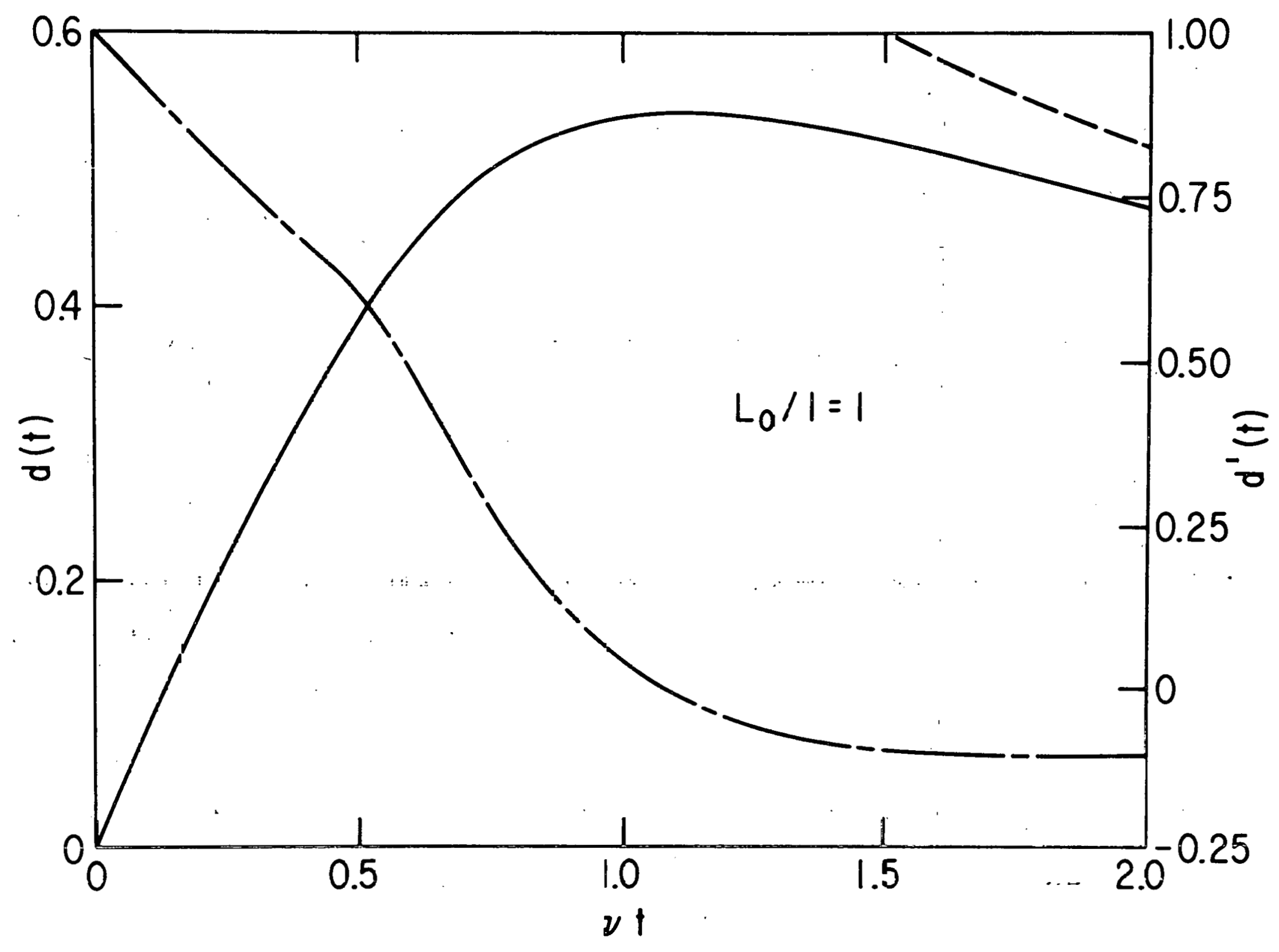

Fig. 2. 782083 


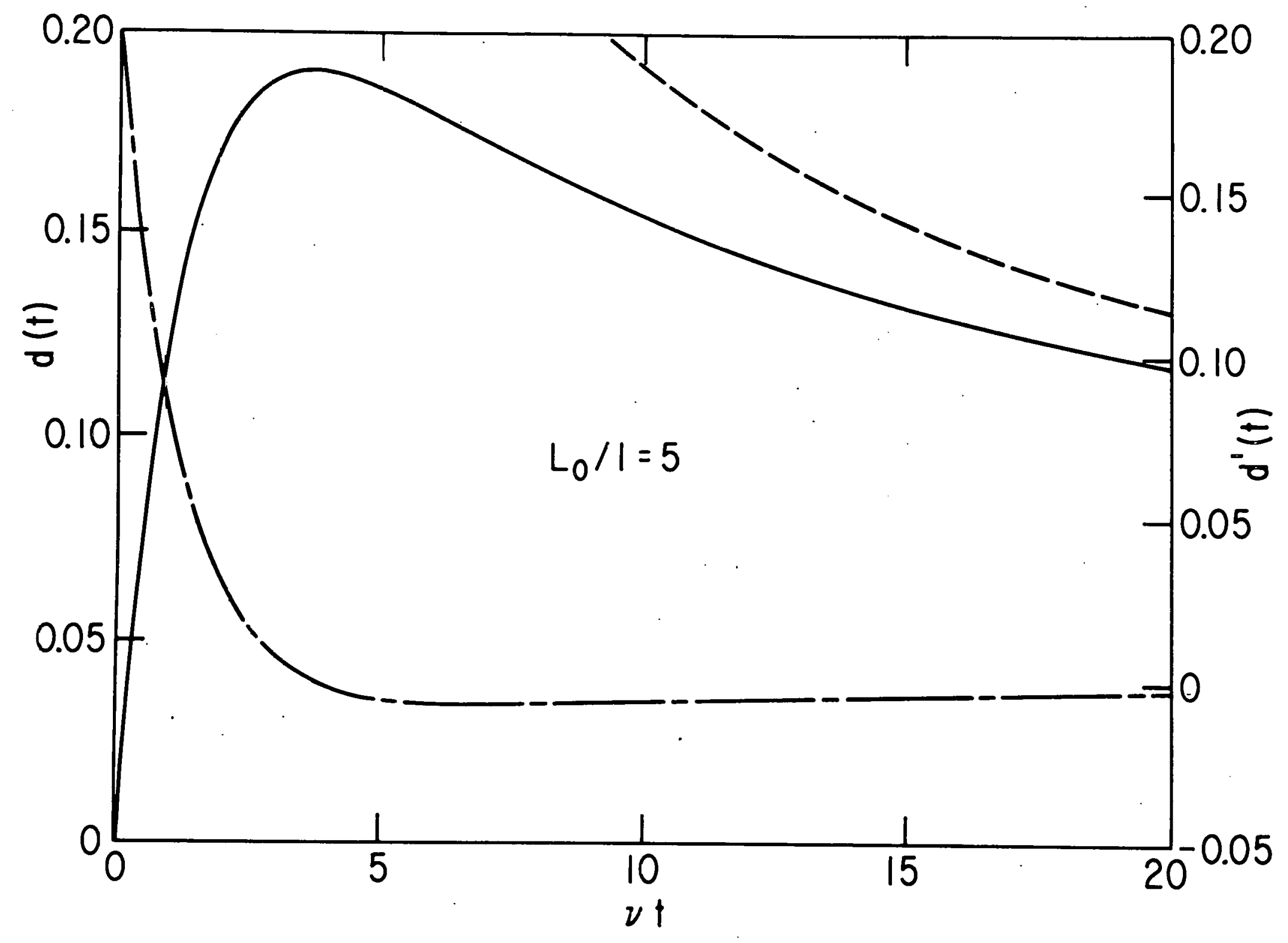

Fig. 3. 782084 
(a)

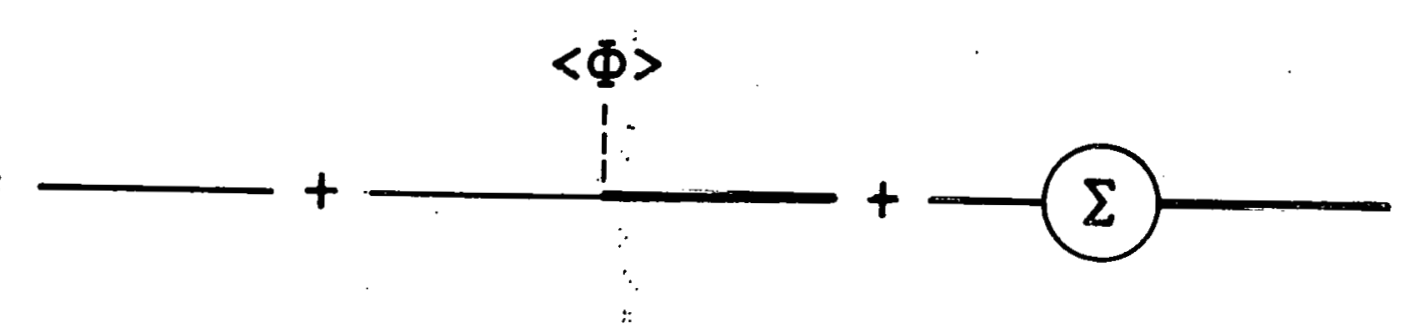

(b)

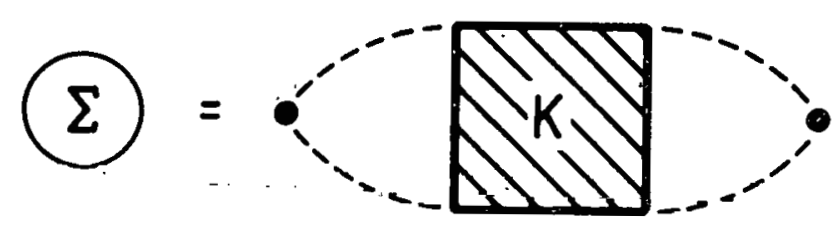

(c)

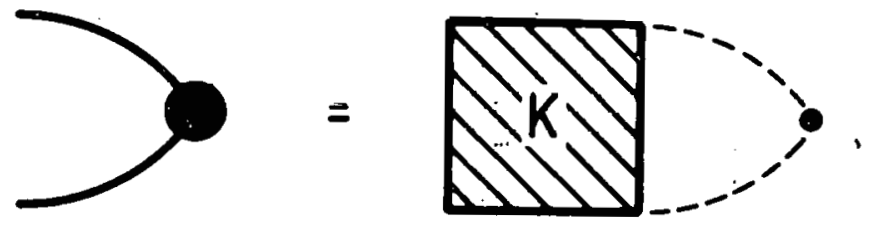

(d)

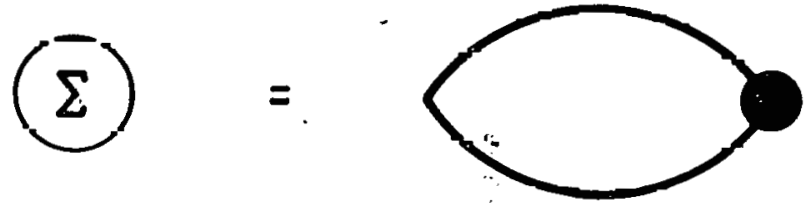

(e)

(E) $\simeq$

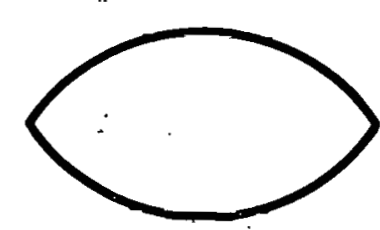

Fig. 4. 782080 


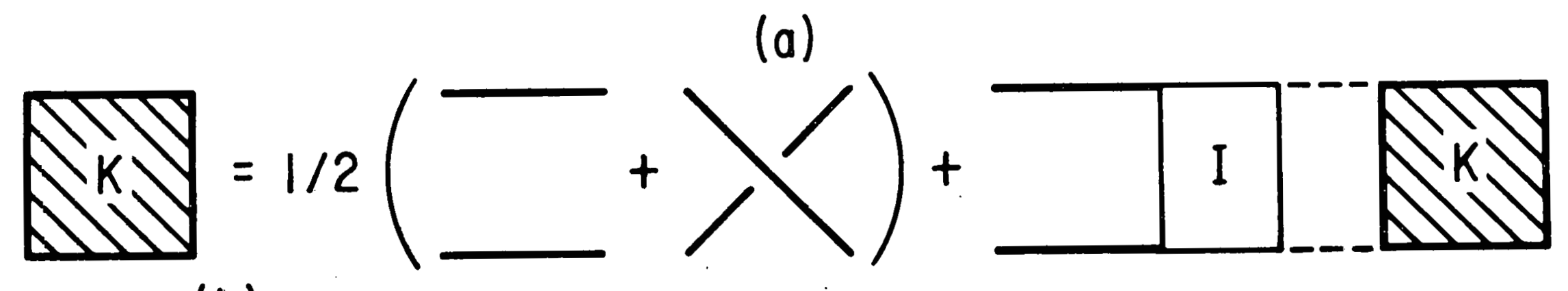

(b)

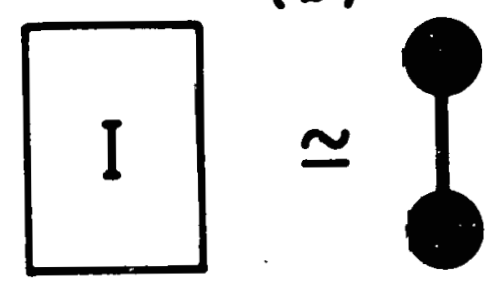

(c)
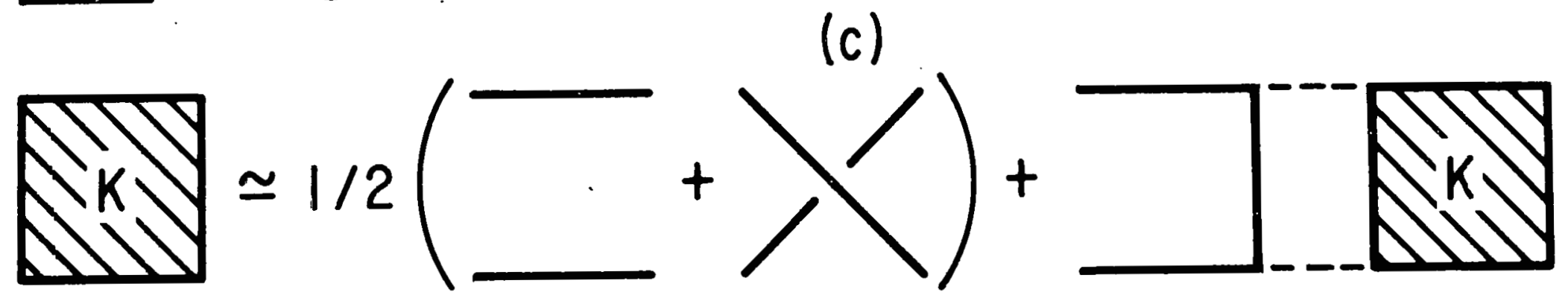

Fig. 5. 782086 
(a)

$F\left(1,1^{\prime}\right) \equiv ' \bigwedge$

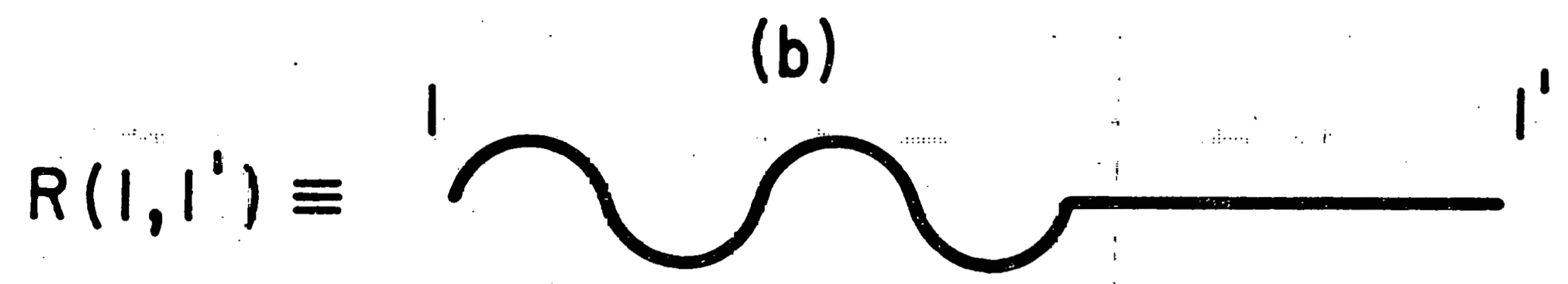

Fig. 6. 782085 


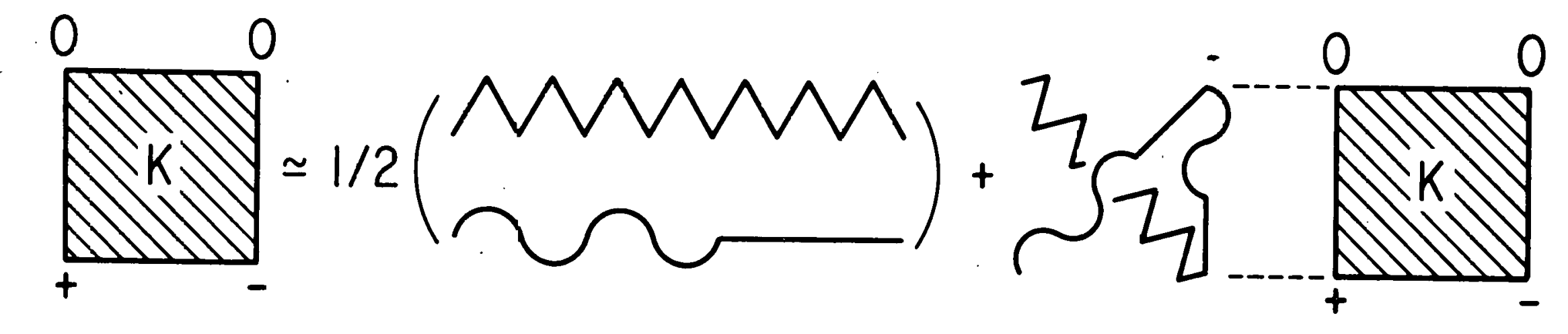




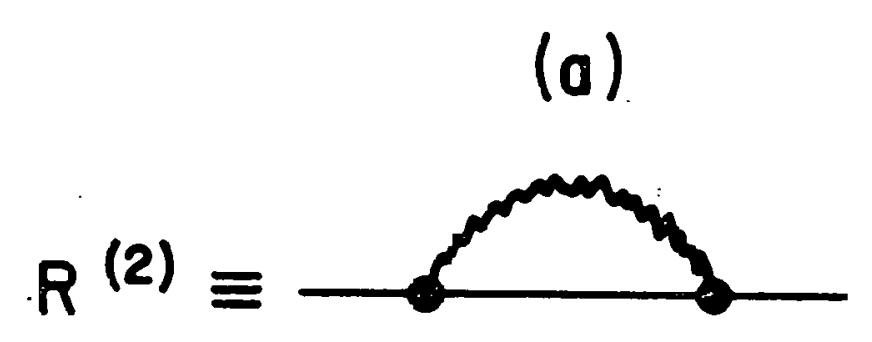

(b)

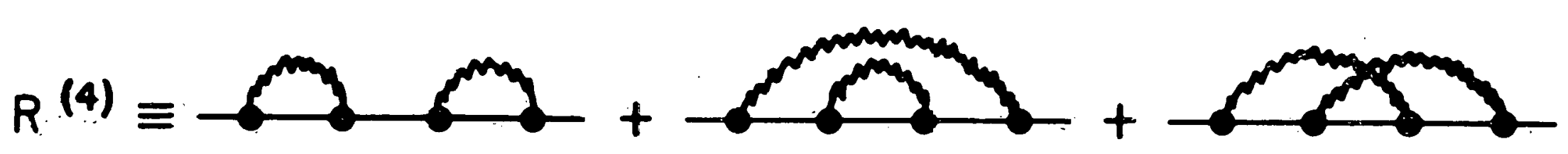




\section{(a)}

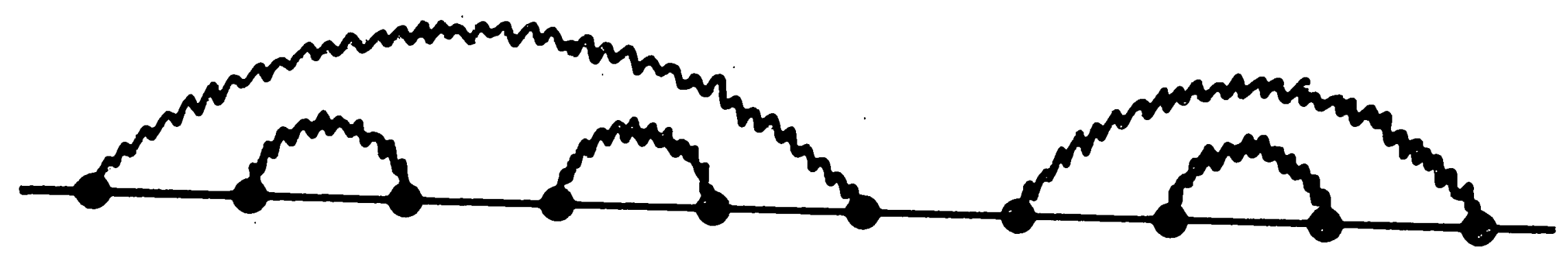

(b)

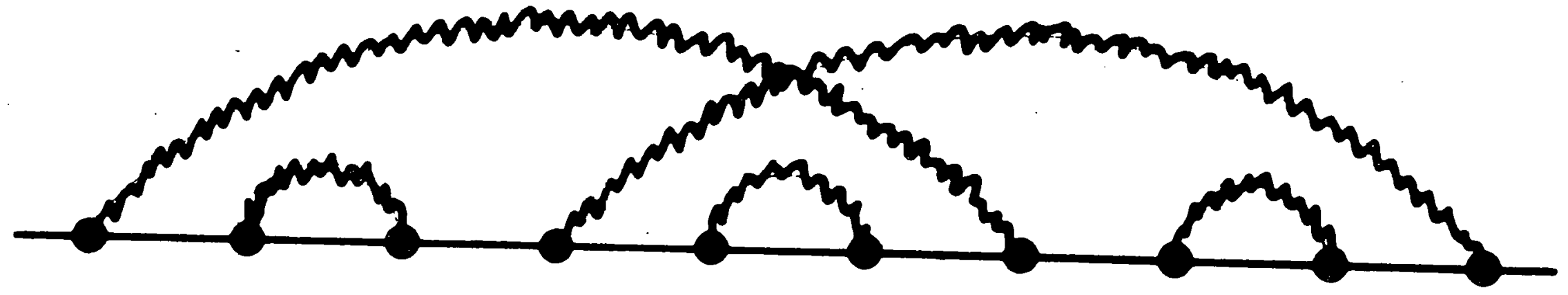

Fig. 9. 782081 